\title{
REGULAR CARDINALS IN MODELS OF ZF
}

BY

MOTI GITIK

\begin{abstract}
We prove the following
THEOREM. Suppose $M$ is a countable model of $Z F C$ and $\kappa$ is an almost huge cardinal in $M$. Let $A$ be a subset of $\kappa$ consisting of nonlimit ordinals. Then there is a model $N_{A}$ of $Z F$ such that $\aleph_{\alpha}$ is a regular cardinal in $N_{A}$ iff $\alpha \in A$ for every $\alpha>0$.
\end{abstract}

0. Introduction. We consider the following question. What are the restrictions in ZF on the class of all regular cardinals? Clearly, $\boldsymbol{\kappa}_{0}$ is always regular and $\boldsymbol{\aleph}_{\omega}, \boldsymbol{\kappa}_{\omega+\omega}$, $\boldsymbol{\aleph}_{\omega^{\omega}}$ and $\boldsymbol{\aleph}_{\alpha}$, for $\alpha$ the least s.t. $\boldsymbol{\aleph}_{\alpha}=\alpha$, are singular. For a limit $\alpha$, if $\boldsymbol{\aleph}_{\alpha}$ is regular, then it is already quite large and its existence is unprovable in ZF. In ZFC, $\aleph_{\alpha+1}$ is a regular cardinal for every $\alpha$. Feferman and Levy [3] proved that it is not true in ZF alone. They built a model of $\mathrm{ZF}+$ " $\boldsymbol{N}_{1}$ is singular". Combining their method with Easton's work [2], it is possible to build a model of ZF + "for every $\alpha, \aleph_{\alpha}$ or $\aleph_{\alpha+1}$ is singular". Now to make both $\aleph_{\alpha}$ and $\aleph_{\alpha+1}$ some additional assumptions are needed.

Thus, the Dodd and Jensen Covering Lemma [1] implies that at least $0^{\dagger}$ is needed, and by Mitchell [11] it looks like at least a hypermeasurable cardinal is needed. On the other hand, a model of $\mathrm{ZF}+$ " $\forall \alpha>0 \aleph_{\alpha}$ is a singular cardinal" is constructed in [5] from the much stronger assumption $\operatorname{Con}\left(\mathrm{ZFC}+{ }^{\prime \prime} \forall \alpha \exists \kappa>\alpha \kappa\right.$ is strongly compact"). The simplest question, which is unclear how to handle by methods of [5], is the following. Is there a model of ZF s.t. those regular alephs are only $\boldsymbol{\aleph}_{0}$ and $\boldsymbol{\aleph}_{1}$ ? Or, for example, let $A=\{\alpha+120 \mid \alpha \in 0 n\}$. Is there a model of ZF s.t. those regular alephs are exactly $\left\{\boldsymbol{\kappa}_{\alpha} \mid \alpha \in A \cup\{0\}\right\}$, i.e., $\boldsymbol{\aleph}_{0}, \boldsymbol{\aleph}_{120}, \boldsymbol{\aleph}_{240}, \ldots$ ?

We shall prove the following

THEOREM. Suppose $M$ is a countable model of ZFC and $\kappa$ is an almost huge cardinal in $M$. Let $A$ be a subset of $\kappa$ consisting of nonlimit ordinals. Then there is a model $N_{A}$ of $Z F$ s.t. those regular alephs are exactly $\left\{\boldsymbol{\kappa}_{\alpha} \mid \alpha \in A \cup\{0\}\right\}$.

As an immediate consequence we obtain

THEOREM. If $Z F C+(\exists \kappa)(\kappa$ is an almost huge cardinal) is consistent, then there is a model $M$ of $Z F C$ s.t. for every class $A$ of $M$ consisting of nonlimit ordinals there exists a model $N_{A}$ of $Z F$ s.t. those regular alephs are exactly $\left\{\boldsymbol{N}_{\alpha} \mid \alpha \in A \cup\{0\}\right\}$.

Notice, that for $A=\varnothing$ it gives a model with all uncountable $\aleph_{\alpha}$ 's singular, but the assumption here is stronger than those of [5].

Received by the editors July 6, 1983.

1980 Mathematics Subject Classification. Primary 04E03; Secondary 03E35, 03E46. 
The proof of our main theorem uses the combination of Levy, Prikry and Radin forcing techniques.

We refer to Jech's book [7] for most terminology and notation, to [9 or 15] for large cardinals used here, and to [4, 12 and 14] for the Radin forcing. Let us summarise here only the more used notions.

$0 n$ denotes the class of all ordinals. For a set $A$ and a cardinal $\kappa, \mathscr{P}_{\kappa}(A)$ is the set of all subsets of $A$ having cardinality less than $\kappa$. For $P \in \mathscr{P}_{\kappa}(A), o(P)$ denotes the order type of $P$, if $A$ is a set of ordinals. For an ordinal $\lambda \geqslant \kappa$ and $n \in \omega$, let $\left[\mathscr{P}_{\kappa}(\lambda)\right]^{n}$ be the set of all the sequences $\left\langle Q_{1}, \ldots, Q_{n}\right\rangle$ such that $Q_{j} \in \mathscr{P}_{\kappa}(\lambda), Q_{j} \cap \kappa$ is an ordinal and $O\left(Q_{j^{\prime}}\right)<Q_{j} \cap \kappa$ for every $j^{\prime}<j, 1 \leqslant j^{\prime}, j \leqslant n$. Let $\left[\mathscr{P}_{k}(\lambda)\right]^{<\omega}=$ $\cup_{n \in \omega}\left[\mathscr{P}_{k}(\lambda)\right]^{n}$. A filter $\mathscr{F}$ over $\mathscr{P}_{\kappa}(A)$ is fine if $\{P \mid a \in P\} \in \mathscr{F}$ for every $a \in A$. F is normal if, for every set of positive measure $B$ and every choice function $F$ on $B$ (i.e. a function $F$ such that $F(P) \in P$ for every $P \in B-\{0\}$ ), there exists $B^{\prime} \subseteq B$, where $B^{\prime}$ is of positive measure such that $F$ is constant on $B^{\prime}$. For a cardinal $\lambda \geqslant \kappa, \kappa$ is $\lambda$-supercompact if there exists a $\kappa$-complete, fine and normal ultrafilter on $\mathscr{P}_{\kappa}(\lambda)$. $\kappa$ is supercompact if $\kappa$ is $\lambda$-supercompact for every cardinal $\lambda \geqslant \kappa$. $\kappa$ is $<\lambda$ supercompact if $\kappa$ is $\beta$-supercompact for every cardinal $\beta<\lambda$. $\kappa$ is an almost huge cardinal if there is an elementary embedding of $V$ into $M$, where $M$ is a transitive class s.t. $j(\alpha)=\alpha$ for $\alpha<\kappa, j(\kappa)>\kappa$ and $M$ is closed under sequences of its elements of length $<j(\kappa)$.

1. The forcing notion. Suppose that $\kappa$ is an almost huge cardinal in a countable model $M$ of ZFC. Let $j: M \rightarrow N$ be an elementary embedding so that $\kappa$ is the first ordinal moved and $N$ is closed under $<j(\kappa)$-sequences of its elements. For every cardinal $\alpha, \kappa \leqslant \alpha<j(\kappa)$, we can define a normal measure $\mu$ on $\mathscr{P}_{\kappa}(\alpha)$ by setting $\mu(X)=1$ iff $j^{\prime \prime}(\alpha) \in j(X)$. Hence $\kappa$ is $<j(\kappa)$-supercompact and the same is true in $N$ since it is closed enough to include all such measures. Let us consider the set of all ordinals $\alpha<\kappa$ which are $<\kappa$-supercompact. By elementarity of $j$, this set is unbounded in $\kappa$. Hence its image under $j$ is unbounded in $j(\kappa)$. For a cardinal $\alpha<\kappa$ (or $\kappa \leqslant \alpha<j(\kappa)$ ) we shall denote by $\alpha^{*}$ the least $<\kappa$-supercompact (or $<j(\kappa)$ supercompact) cardinal above $\alpha$.

Let $\alpha<\beta<\kappa$ be regular cardinals. We shall denote by $\operatorname{Col}(\alpha, \beta)$ the Levy collapse of all cardinals below $\beta$ to $\alpha$, i.e., $\operatorname{Col}(\alpha, \beta)=\{f \mid f$ is a partial function from $\beta \times \alpha$ into $\beta$, s.t. $|f|<\alpha$ and, for every $\gamma_{1}<\beta, \gamma_{2}<\alpha$ in the domain of $f$, $\left.f\left(\gamma_{1}, \gamma_{2}\right)<\gamma_{1}\right\}$. The order on $\operatorname{Col}(\alpha, \beta)$ is the inclusion.

Suppose now that $\alpha$ is $\beta$-supercompact.

We shall denote by $\mathscr{P}(\alpha, \beta)$ the supercompact Prikry forcing on $\mathscr{P}_{\alpha}(\beta)$, i.e., $\mathscr{P}(\alpha, \beta)$ is the set of all finite sequences $\left\langle Q_{1}, \ldots, Q_{n}, B\right\rangle$, where $\left\langle Q_{1}, \ldots, Q_{n}\right\rangle \in$ $\left[\mathscr{P}_{\alpha}(\beta)\right]^{n}, Q_{i} \cap \kappa$ is a cardinal for $1 \leqslant i \leqslant n, B$ is a set of measure one for some fixed $\alpha, \beta$ normal measure on $\mathscr{P}_{\alpha}(\beta)$, and, for every $Q \in B$ and $Q \supseteq Q_{n}, Q \cap \kappa$ is a cardinal and $Q \cap \kappa>O\left(Q_{n}\right)$, where $O\left(Q_{n}\right)$ is the order type of $Q_{n}$. Let $\left\langle Q_{i 1}, \ldots, Q_{\text {in }_{i}}, B_{i}\right\rangle \in \mathscr{P}(\alpha, \beta), i \in 2$. Then $\left\langle Q_{01}, \ldots, Q_{0 n_{0}}, B_{0}\right\rangle \leqslant\left\langle Q_{11}, \ldots, Q_{1 n_{1}}, B_{1}\right\rangle$ if

(a) $n_{0} \leqslant n_{1}$,

(b) $\left\langle Q_{01}, \ldots, Q_{0 n_{0}}\right\rangle=\left\langle Q_{11}, \ldots, Q_{1 n_{0}}\right\rangle$, 
(c) $B_{1} \subseteq B_{0}$, and

(d) $\left\langle Q_{1 n_{0}+1}, \ldots, Q_{1 n_{1}}\right\rangle \in\left[B_{0}\right]^{n_{1}-n_{0}}$.

Notice that for $\alpha=\beta$, it is the usual Prikry forcing. More involved forcing notion of such kind was used by Magidor in [10].

Using the almost huge embedding $j$ let us define by induction a sequence of measures $M_{<\kappa^{+}}=\left\langle\mu_{\beta} \mid \beta<\kappa^{+}\right\rangle$of length $\kappa^{+}$on $\mathscr{P}_{\kappa}\left(\kappa^{*}\right) \times V_{\kappa}$ as follows:

(1) $\mu_{0}(X)=1$ if $\left\langle j^{\prime \prime}\left(\kappa^{*}\right), \varnothing\right\rangle \in j(X)$.

(2) $\mu_{\beta}(X)=1$ if $\left\langle j^{\prime \prime}\left(\kappa^{*}\right),\left\langle\mu_{\beta^{\prime}} \mid \beta^{\prime}<\beta\right\rangle\right\rangle \in j(X)$.

Let $\mathbf{R}$ be the supercompact Radin forcing with $M_{<\kappa^{+}}$. We refer to $[4,14]$ for the detailed definitions. Let us only mention how a condition in $\mathbf{R}$ looks. It is a finite sequence $\left\langle\left\langle P_{0}, u_{0}, B_{0}\right\rangle, \ldots,\left\langle P_{n}, u_{n}, B_{n}\right\rangle,\left\langle M_{\left\langle\kappa^{+}\right.}, B\right\rangle\right\rangle$, where $\left\langle P_{0}, \ldots, P_{n}\right\rangle \in$ $\left[\mathscr{P}_{\kappa}\left(\kappa^{*}\right)\right]^{n}, B$ is a sequence of sets of measure one for $M_{<\kappa^{+}}$, and, for every $i$, $1 \leqslant i \leqslant n$, the following hold:

(1) $P_{i} \cap \kappa$ is a cardinal.

(2) $\left(P_{i} \cap \kappa\right)^{*}=O\left(P_{i}\right)$, i.e., $O\left(P_{i}\right)$ is the least $<\kappa$-supercompact above $P_{i} \cap \kappa$.

(3) $u_{i}$ is a sequence of measures over $\mathscr{P}_{P_{i} \cap \kappa}\left(\left(P_{i} \cap \kappa\right)^{*}\right) \times V_{P_{i} \cap \kappa}$, or $u_{i}=\varnothing$ and then we shall omit it often.

(4) $B_{i}$ is a sequence of sets of measure one for the sequence of measures $u_{i}$.

Finally let $\mathbf{P}$ be the finite support iteration of $\mathbf{R}, \operatorname{Col}(\alpha, \beta)$ and $\mathscr{P}(\gamma, \delta)$ for all regular cardinals $\alpha, \beta, \gamma, \delta<\kappa$ s.t. $\alpha \leqslant \beta$ and $\gamma$ is $\delta$-supercompact.

Clearly, if we force with $\mathbf{P}$, then everything below $\boldsymbol{\kappa}^{+}$is collapsed to $\omega$. But we are interested in some submodels of a generic extension of $\mathbf{P}$.

2. The group of automorphisms. Let us separately define groups of automorphisms of $\operatorname{RO}(\operatorname{Col}(\gamma, \delta)), \operatorname{RO}(\mathscr{P}(\alpha, \beta))$ and $\operatorname{RO}(\mathbf{R})$ for every regular $\alpha, \beta, \gamma, \delta$ s.t. $\gamma \leqslant \delta$ and $\alpha$ is $\beta$-supercompact. (RO stands for regular open.) Then taking their direct product, we shall define the group of automorphisms of $\operatorname{RO}(P)$.

First let $\gamma<\delta \leqslant \kappa$ be regular ordinals. For every set $\bar{B}=\left\{\left\langle\beta, \pi_{\beta}\right\rangle \mid \beta \in B\right\}$ s.t. $B$ is a set of ordinals between $\gamma$ and $\delta$ of cardinality less than $\gamma$ and, for every $\beta \in B$, $\pi_{\beta}$ is a permutation of $\beta$ which moves less than $\gamma$ ordinals, we define an automorphism $\pi_{\bar{B}}$ of $\operatorname{Col}(\gamma, \delta)$ as follows. $\pi_{\bar{B}}(p)=q$, where $\left\langle\xi, \nu_{1}, \nu_{2}\right\rangle \in q$ iff

(1) $\xi \notin B$ and $\left\langle\xi, \nu_{1}, \nu_{2}\right\rangle \in p$,

(2) $\xi \in B$ and, for some $\nu_{3}<\xi$ s.t. $\pi_{\xi}\left(\nu_{3}\right)=\nu_{2}\left\langle\xi, \nu_{1}, \nu_{3}\right\rangle \in p$.

Let $\mathscr{G}_{1}(\gamma, \delta)$ be the group that consists of such $\pi_{\bar{B}}$ 's.

Such automorphisms were used by Levy [8] in the construction of a model of ZF with $\aleph_{1}$ of countable cofinality.

Now let us define automorphisms for the forcing $\mathscr{P}(\alpha, \beta)$. Let $\mathscr{U}$ be the fixed normal ultrafilter over $\mathscr{P}_{\alpha}(\beta)$. Let $p=\left\langle P_{1}, \ldots, P_{n}, A\right\rangle$ and $q=\left\langle Q_{1}, \ldots, Q_{N}, A\right\rangle$ be elements of $\mathscr{P}(\alpha, \beta)$. Our aim is to define an automorphism $\pi_{p, q}$ of $\operatorname{RO}(P(\alpha, \beta))$ which takes $p$ to $q$. It is enough to define $\pi_{p, q}$ only on a dense subset of $\mathscr{P}(\alpha, \beta)$. Let $D=\{r \in \mathscr{P}(\alpha, \beta) \mid r \geqslant p$ or $r \geqslant q$ or $r$ is incompatible with both $p$ and $q\}$. Clearly $D$ is a dense open subset of $\mathscr{P}(\alpha, \beta)$. We define $\pi_{p, q}$ on $D$. Let $r \in D$. If $r$ is incompatible with both $p$ and $q$, then let $\pi_{p, q}(r)=r$. Otherwise $r \geqslant p$ or $r \geqslant q$. Let us assume that $r \geqslant p$. In case $r \geqslant q$ the definition is the same except that $P$ 's must 
be replaced by $Q$ 's. There are $P_{N+1}, \ldots, P_{N+m} \in A$ and $A^{\prime} \subseteq A, A^{\prime} \in \mathscr{U}$ s.t. $r=$ $\left\langle P_{1}, \ldots, P_{N}, P_{N+1}, \ldots, P_{N+m}, A^{\prime}\right\rangle$. Set $\pi_{p, q}(r)=\left\langle Q_{1}, \ldots, Q_{n}, P_{N+1}, \ldots, P_{N+m}, A^{\prime}\right\rangle$. Then, clearly, $\pi_{p, q}(r) \in \mathscr{P}(\alpha, \beta), \pi_{p, q}(r) \geqslant q$ and so it is in $D$.

Such defined $\pi_{p, q}$ is an automorphism of $D$ since it is one-to-one and preserves the order. Let $\mathscr{G}_{2}(\alpha, \beta)$ be the group of automorphisms of $\operatorname{RO}(\mathscr{P}(\alpha, \beta))$ generated by all such $\pi_{p, q}$, i.e., where $p, q \in \mathscr{P}(\alpha, \beta)$ are of the same length and have the same set of measure one. We shall call $p, q$ as above similar conditions.

Finally let us define a group of automorphisms $\mathscr{G}_{3}$ of $\mathbf{R O}(\mathbf{R})$ for the supercompact Radin forcing $\mathbf{R}$. The construction of $\mathscr{G}_{3}$ will be similar to $\mathscr{G}_{2}(\alpha, \beta)$.

For an element $P$ of $\mathscr{P}_{\kappa}\left(\kappa^{*}\right)$, let $C_{P}: P \leftrightarrow O(P)$ be the transitive collapse of $P$.

Definition 2.0. Let $p=\left\{\left\langle P_{1}, u_{1}, A_{1}\right\rangle, \ldots,\left\langle P_{N}, u_{N}, A_{N}\right\rangle,\left\langle M_{<\kappa^{+}}, A\right\rangle\right\}$ and $q=$ $\left\{\left\langle Q_{1}, v_{1}, B_{1}\right\rangle, \ldots,\left\langle Q_{N^{\prime}}, v_{N^{\prime}}, B_{N^{\prime}}\right\rangle,\left\langle M_{<\kappa^{+}}, B\right\rangle\right\}$ be two elements of $\mathbf{R}$. Suppose that for every $i, 1 \leqslant i \leqslant N$ :

(1) $N=N^{\prime}$,

(2) $A=B$,

(3) $u_{i}=\varnothing$ iff $v_{i}=\varnothing$, and

(4) $u_{i} \neq \varnothing$ implies that

(a) $P_{i} \cap \kappa=Q_{i} \cap \kappa\left(\right.$ and hence $\left.O\left(P_{i}\right)=\left(P_{i} \cap \kappa\right)^{*}=\left(Q_{i} \cap \kappa\right)^{*}=O\left(Q_{i}\right)\right)$, and (b) $\left\langle V_{i}, B_{i}\right\rangle=\left\langle u_{i}, A_{i}\right\rangle$.

Then we say that $p$ and $q$ are similar.

We define an automorphism $\pi_{p, q}$ on a dense set $D=\{r \in \mathbf{R} \mid r \geqslant p$ or $r \geqslant q$ or $r$ is incompatible with both $p$ and $q$ \} for $p, q$ similar elements of $\mathbf{R}$. If $r$ is incompatible with $p$ and $q$, then let $\pi_{p, q}(r)=r$. Otherwise suppose that $r \geqslant p(r \geqslant q$ is similar $)$. Let

$$
p=\left\{\left\langle P_{1}, u_{1}, A_{1}\right\rangle, \ldots,\left\langle P_{N}, u_{N}, A_{N}\right\rangle,\left\langle M_{<\kappa^{+}}, A\right\rangle\right\}
$$

and

$$
q=\left\{\left\langle Q_{1}, u_{1}, A_{1}\right\rangle, \ldots,\left\langle Q_{N}, u_{N}, A_{N}\right\rangle,\left\langle M_{<\kappa^{+}}, A\right\rangle\right\}
$$

Then $r$ looks like $\left\{\left\langle P_{11}, u_{11}, A_{11}\right\rangle, \ldots,\left\langle P_{1 n_{1}}, u_{1 n_{1}}, A_{1 n_{1}}\right\rangle,\left\langle P_{1}, u_{1}, A_{1}^{\prime}\right\rangle,\left\langle P_{21}, u_{21}\right.\right.$, $\left.A_{21}\right\rangle, \ldots,\left\langle P_{N n_{N}}, u_{N n_{N}}, A_{N n_{N}}\right\rangle,\left\langle P_{N}, u_{N}, A_{N}^{\prime}\right\rangle,\left\langle P_{N+1}, u_{N+1}, A_{N+1}\right\rangle, \ldots$, $\left.\left\langle P_{N+m}, u_{N+m}, A_{N+m}\right\rangle,\left\langle M_{<\kappa^{+}}, A^{\prime}\right\rangle\right\}$, where $n_{i}$ and $m$ can be equal to 0 .

Set $\pi_{p, q}(r)=\left\{\left\langle Q_{11}, u_{11}, A_{11}\right\rangle, \ldots,\left\langle Q_{1 n_{1}}, u_{1 n_{1}}, A_{1 n_{1}}\right\rangle,\left\langle Q_{1}, u_{1}, A_{1}^{\prime}\right\rangle,\left\langle Q_{21}, u_{21}\right.\right.$, $\left.A_{21}\right\rangle, \ldots,\left\langle Q_{N n_{N}}, u_{N n_{N}}, A_{N n_{N}}\right\rangle,\left\langle Q_{N}, u_{N}, A_{N}^{\prime}\right\rangle, \ldots,\left\langle P_{N+1}, u_{N+1}, A_{N+1}\right\rangle, \ldots$, $\left.\left\langle P_{N+m}, u_{N+m}, A_{N+m}\right\rangle,\left\langle M_{<\kappa^{+}}, A^{\prime}\right\rangle\right\}$, where, for every $i, j$ s.t. $1 \leqslant i \leqslant N, 1 \leqslant j \leqslant n_{i}$ and $u_{i} \neq \varnothing$,

$$
Q_{i j}=C_{Q_{i}}^{-1} \circ C_{P_{i}}^{\prime \prime}\left(P_{i j}\right)
$$

Notice that $p$ and $q$ are similar and hence by Definition 2.0(4)(a), $O\left(P_{i}\right)=O\left(Q_{i}\right)$. So $C_{Q_{i}}^{-1} \circ C_{P_{i}}$ is well defined.

LEMMA 2.1. $\pi_{p, q}$ is an automorphism of $D$. 
Proof. First of all let us check that if $r \geqslant p$, then $\pi_{p, q}(r) \in D$ and it is stronger than $q$. Let $1 \leqslant i \leqslant N$. If $n_{i} \neq 0$, then let us show that

$$
\left\{\left\langle Q_{i 1}, u_{i 1}, A_{i 1}\right\rangle, \ldots,\left\langle Q_{i n_{i}}, u_{i n_{i}}, A_{i n_{i}}\right\rangle\right\}
$$

is addable to $q$. Notice that $n_{i} \neq 0$ implies $u_{i} \neq \varnothing$. Suppose that $n_{i} \neq 0$ and $1 \leqslant j \leqslant n_{i}$. Then $C_{P_{i}}^{\prime \prime}\left(P_{i j}\right)=C_{Q_{i}}^{\prime \prime}\left(Q_{i j}\right)$ is in $A_{n_{i}}$. Since $q \in \mathbf{R}$, it implies that $Q_{i j} \supseteq$ $Q_{i-1}, Q_{i j} \cap \kappa>Q_{i-1} \cap \kappa$ (if $i \geqslant 2$ ). Now since $\left\{\left\langle P_{i j}, u_{i j}, A_{i j}\right\rangle \mid 1 \leqslant j \leqslant n_{i}\right\}$ is addable to $p,\left\{\left\langle Q_{i j}, u_{i j}, A_{i j}\right\rangle \mid 1 \leqslant j \leqslant n_{i}\right\}$ is addable to $q$.

So for every $i, 1 \leqslant i \leqslant N, n_{i} \neq 0$ implies that $\left\{\left\langle Q_{i j}, u_{i j}, A_{i j}\right\rangle \mid 1 \leqslant j \leqslant n_{i}\right\}$ is addable to $q$. But then $\pi_{p, q}(r) \in \mathbf{R}$ and $\pi_{p, q}(r) \geqslant q$.

D.

Clearly $\pi_{p, q}$ is one-to-one and order preserving. Hence $\pi_{p, q}$ is an automorphism of

Let $\mathscr{G}_{3}$ be the group of automorphisms of $\mathbf{R O}(\mathbf{R})$ generated by all such $\pi_{p, q}$.

Lastly let $\mathscr{G}$ be the direct product of $\left\{\mathscr{G}_{1}(\alpha, \beta), \mathscr{G}_{2}(\gamma, \delta), \mathscr{G}_{3} \mid \alpha, \beta, \gamma, \delta<\kappa\right.$ regular cardinals, $\alpha \leqslant \beta, \gamma$ is $\delta$-supercompact $\}$. $\mathscr{G}$ is a group of automorphisms of $\operatorname{RO}(\mathbf{P})$.

3. The models. Now let us fix some subset $A$ of $\kappa$ in $M$ consisting of nonlimit ordinals. We are going to define a submodel $N_{A}$ of a generic extension of $\boldsymbol{M}$ by $\mathbf{P}$. For every ordinal $\alpha>0, \aleph_{\alpha}$ will be a regular in $N_{A}$ iff $\alpha \in A$. Our strategy will be as follows. First, we construct a set $A(G)$ in $M[G]$ (where $G$ is now a fixed generic subset of $\mathbf{P}$ ) which contains all the information about the structure of cardinals of $N_{A}$. Second, we consider $\mathrm{H} M \mathrm{D}(A(G))$, the class of all sets hereditarily $M$-definable over $A(G)$; see Jech [7] for the definitions. And finally, we set $N_{A}=$ the set of all elements of $\mathrm{HMD}(A(G))$ of rank less than $\kappa$. Using the fact that the length of the Radin forcing is $\kappa^{+}$, we prove that $N_{A}$ is a model of $\mathrm{ZF}$.

3.1. Construction of $A(G)$. Let us first introduce some notation. For a forcing notion $P$ let $\stackrel{\circ}{P}$ denote a generic subset of $P$. By the Radin sequence we mean the set $R_{s}=\left\{P \in \mathscr{P}_{\kappa}^{M}\left(\kappa^{*}\right) \mid \exists p \in \stackrel{\mathbf{R}}{P}\right.$ appears in $\left.p\right\}$. Let the ordinal part of the Radin sequence be the set $R_{s o}=\left\{\alpha<\kappa \mid \exists P \in R_{s} P \cap \kappa=\alpha\right\}$. Notice that both $R_{s}$ and $R_{s o}$ are increasing and continuous. $R_{\text {so }}$ is unbounded in $\kappa$ and for every $P \in \mathscr{P}_{\kappa}^{M}\left(\kappa^{*}\right)$ there is $Q \in R_{s}, Q \supseteq P$. Let $R_{s l}=\left\{P \in R_{s} \mid P\right.$ is a limit point of $\left.R_{s}\right\}$ and $R_{s o l}=\{\alpha$ $<\kappa \mid \alpha$ is a limit point of $R_{s o}$.

The class of all cardinals of $N_{A}$ will be equal to the set $E=\left\{\alpha<\kappa \mid \alpha \in R_{\text {sol }}\right.$ or $\alpha=\beta^{*}$ for an element $\beta$ of $\left.R_{\text {sol }}\right\} \cup\left\{\omega, \omega^{*}\right\}$. Let $\left\langle\alpha_{\nu} \mid \nu<\kappa\right\rangle$ be the increasing continuous enumeration of $E$. Then in $N_{A}$ we shall have $\aleph_{\nu}=\alpha_{\nu}(\nu<\kappa)$. In order to insure that $N_{A} \vDash \boldsymbol{N}_{\nu}$ regular iff $\nu \in A$, let us do the following. If $\nu \in A$ or $\nu=0$, then we add to $A(G)$ the information about the Levy collapse between $\alpha_{\nu}$ and $\alpha_{\nu+1}$. Otherwise we shall add to $A(G)$ information from the supercompact Radin forcing or supercompact Prikry forcing about collapsing cardinals between $\alpha_{\nu}$ and $\alpha_{\nu+1}$, namely, if $\nu=\nu^{\prime}+2 k+1$, then add from the Prikry forcing, else from the Radin forcing (where $\nu^{\prime}$ is the maximal limit ordinal $\leqslant \nu$ and $k \in \omega$ ).

Now let us do the above formally. Suppose an ordinal $\nu$ is less than $\kappa$ and $\beta$ is a cardinal in the interval $\left[\alpha_{\nu}, \alpha_{\nu+1}\right)$. Let $\nu=\nu^{\prime}+n$, where $\nu^{\prime}$ is a limit ordinal and $n \in \omega$. We consider few cases. 
(1) $\nu^{\prime}>0$ and $n=0$. Define

$$
\stackrel{\circ}{R}_{s}\left(\alpha_{\nu}, \beta\right)=\left\{C_{P\left(\alpha_{\nu}\right)}^{\prime \prime}(P) \cap \beta \mid P \in R_{s l}, P \subseteq P\left(\alpha_{\nu}\right) \text { and } C_{P\left(\alpha_{\nu}\right)}^{-1}(\beta) \in P\right\},
$$

where $P\left(\alpha_{\nu}\right)$ is the element of $R_{s}$ s.t. $P\left(\alpha_{\nu}\right) \cap \kappa=\alpha_{\nu}$ and $C_{P\left(\alpha_{\nu}\right)}: P\left(\alpha_{\nu}\right) \leftrightarrow O\left(P\left(\alpha_{\nu}\right)\right)$ is the transitive collapse of $P\left(\alpha_{\nu}\right)$.

(2) $n=2 k$ for some $k \in \omega-\{0\}$. Define

$$
\begin{aligned}
\stackrel{\circ}{R}_{s}\left(\alpha_{\nu}, \beta\right)= & \left\{C_{P\left(\alpha_{\nu}\right)}^{\prime \prime}(P) \cap \beta \mid P \in R_{s}\right. \text { and if } \\
& \left.\nu^{\prime} \neq 0 \text { or }\left(\nu^{\prime}=0 \text { and } k>1\right), \text { then } P\left(\alpha_{\nu^{\prime}+2(k-1)}\right) \varsubsetneqq P \subseteq P\left(\alpha_{\nu}\right)\right\} .
\end{aligned}
$$

(Notice that in this case all $P$ 's except $P\left(\alpha_{\nu}\right)$ are nonlimit points of $R_{s}$. In the previous case, only limit $P$ 's, i.e., members of $R_{s l}$, are considered.)

(3) $n=2 k+1$ for some $k \in \omega-\{0\}$. Define

$$
\stackrel{\circ}{\mathscr{P}}_{s}\left(\alpha_{\nu}, \beta\right)=\left\{P \cap \beta \mid \text { there is } p \in \stackrel{\mathscr{P}}{ }\left(\alpha_{\nu}, \alpha_{\nu+1}\right) \text { s.t. } P \text { appears in } p\right\} .
$$

(4) $n \neq 0$ or $n=0$ and $\nu^{\prime}=0$. Define

$$
\operatorname{Coll}\left(\alpha_{\nu}, \beta\right)=\left\{P \uparrow \beta \mid p \in \operatorname{Col}\left(\alpha_{\nu}, \alpha_{\nu+1}\right)\right\} .
$$

Now define $A^{\prime}(G)$ to be the set $\left\{x_{\nu}(\beta) \mid \nu<\kappa, \beta\right.$ is a cardinal s.t. $\left.\alpha_{\nu} \leqslant \beta<\alpha_{\nu+1}\right\}$, where:

(a) if $\nu \neq 0$ is limit, then $x_{\nu}(\beta)=\stackrel{\circ}{R}_{s}\left(\alpha_{\nu}, \beta\right)$,

(b) if $\nu \in A \cup\{0\}$, then $x_{\nu}(\beta)=\operatorname{Col}\left(\alpha_{\nu}, \beta\right)$,

(c) if $\nu$ is nonlimit and $\nu \notin A$, then, say, $\nu=\nu^{\prime}+n$ for a limit ordinal $\nu^{\prime}$ and $n \in \omega-\{0\}$;

(1) if $n=2 k$, then $x_{\nu}(\beta)=\stackrel{\circ}{R}_{s}\left(\alpha_{\nu}, \beta\right)$,

(2) if $n=2 k+1$, then $x_{\nu}(\beta)=\mathscr{\mathscr { P }}_{s}\left(\alpha_{\nu}, \beta\right)$.

Such defined $A^{\prime}(G)$ already contains all the information we need about collapsing cardinals. The problem is that this set is not symmetric enough to insure that all $\alpha_{\nu}$ remain cardinals in $\mathrm{H} M \mathrm{D}\left(A^{\prime}(G)\right)$. So we shall define a more symmetric set $A(G)$ using $A^{\prime}(G)$.

First let us fix names for elements of $A^{\prime}(G)$. Let us start with the sequence $\left\langle\alpha_{\nu}\right| \nu\langle\kappa\rangle$ and define a canonical name $\underline{\alpha}_{\nu}$ of $\alpha_{\nu}$. Notice that a condition $\{\langle P, u, A\rangle\} \in \mathbf{R}$ forces "the order type of $R_{s}$ below $P$ is equal to

$$
\min \left(P \cap \kappa, \omega^{\text {length }(u)}\right) \text { ". }
$$

Fix an ordinal $\nu, 1<\nu<\kappa$. Let $\omega \cdot \nu=\omega^{\delta_{0}} \cdot m_{0}+\omega^{\delta_{1}} \cdot m_{1}+\cdots+\omega^{\delta_{n}} \cdot m_{n}$, where $\delta_{0}>\cdots>\delta_{n}>0, m_{0}, \ldots, m_{n} \in \omega-\{0\}$ and $+, \cdot, \omega^{\delta}$ are as in the ordinal arithmetics. Set

$$
\begin{aligned}
D(\nu)=\{ & p \in P|p| \mathbf{R} \text { is stronger than some condition } q \\
& =\left\{\left\langle P_{i j_{i}}, u_{i j_{i}}, B_{i j_{i}}\right\rangle \mid i \leqslant n, 1 \leqslant j_{i} \leqslant m_{i}\right\} \cup\left\{\left\langle M_{<\kappa^{+}}, B\right\rangle\right\} \\
& \operatorname{such} \text { that } \min \left(P_{i 1} \cap \kappa, \omega^{\text {length }\left(u_{i 1}\right)}\right)=\delta_{i} \text { and length }\left(u_{i j}\right) \\
& \left.=\min \left(P_{i 1} \cap \kappa, \text { length }\left(U_{i 1}\right)\right) \text { for every } i \leqslant n, 1 \leqslant j \leqslant m_{i}\right\} .
\end{aligned}
$$


Then every element $p$ of $D(\nu)$ knows what the $(\omega \cdot \nu)$ th member of $R_{s}$ is going to be. Namely, for $q$ as above it will be $P_{n m_{n}} \cap \kappa$. Let $\nu=\nu^{\prime}+k$ where $\nu^{\prime}$ is a limit ordinal and $k$ is equal to $m_{n}$ or 0 . Then $p$ determines $\alpha_{\nu^{\prime}+2 k}$. More than that, it determines every $\alpha_{\nu^{\prime}+r}$ for $r \leqslant 2 k+1$. Thus $\alpha_{\nu^{\prime}+2 k}$ will be $P_{n m_{n}} \cap \kappa$ for every $j, 1 \leqslant j \leqslant m_{n}$ (in case $k \neq 0), \alpha_{\nu^{\prime}+2 j}=P_{n j} \cap \kappa$ and $\alpha_{\nu^{\prime}+2 i+1}=\left(\alpha_{\nu^{\prime}+2 i}\right)^{*}$ for every $i \leqslant k$. Clearly different $p$ 's can determine different values for $\alpha_{\nu^{\prime}+r}(r \leqslant 2 k+1)$. So let us denote by $\alpha_{\nu^{\prime}+r}(p) \alpha_{\nu^{\prime}+r}$ determined by $p$.

Also notice that the set $D(\nu)$ is a dense open subset of $\mathbf{P}$.

Now we are ready to define the canonical name $\underline{\alpha}_{\nu}$ of $\alpha_{\nu}$. Set

$$
\underline{\alpha_{\nu}}=\left\{\left\langle p, \alpha_{\nu}^{v}(p)\right\rangle \mid p \in D(\nu)\right\} .
$$

Now let us define canonical names for $\left\langle x_{\nu}(\beta) \mid \beta, \nu<\kappa, \alpha_{\nu} \leqslant \beta<\alpha_{\nu+1}\right\rangle$. Let $\beta$ be a cardinal less that $\kappa$. For a condition $p=\left\{\left\langle P_{0}, u_{0}, B_{0}\right\rangle, \ldots,\left\langle P_{n}, u_{n}, B_{n}\right\rangle\right.$, $\left.\left\langle M_{<\kappa^{+}}, \beta\right\rangle\right\}$ in $\mathbf{R}$ and $\alpha$ s.t. $P_{m} \cap \kappa=\alpha$ for some $m \leqslant n$, let us denote by $p \mid(\alpha, \beta)$ the set $\left\langle C_{P_{m}}^{\prime \prime}\left(P_{i}\right) \cap \beta\right| i \leqslant m, u_{i} \neq \varnothing$ and $\left.\beta \in C_{P_{m}}^{\prime \prime}\left(P_{i}\right)\right\rangle$ if $\alpha \leqslant \beta<\alpha^{*}$, or the set $\left\langle P_{i} \cap \kappa\right| i \leqslant m$ and $\left.u_{i} \neq \varnothing\right\rangle$ otherwise.

Now let $\nu<\kappa$ be an ordinal. Consider the following cases.

Case 1. $\nu \neq 0$ is a limit ordinal. Set

$$
\underline{x_{\nu}(\beta)}=\left\{\left\langle p,(\check{p} \uparrow \mathbf{R}) \uparrow\left(\alpha_{\nu}(p), \beta\right)\right\rangle \mid p \in D(\nu)\right\}
$$

( $\check{p}$ is the canonical name of $p$ ).

Case 2. $\nu \in A \cup\{0\}$. Define

$$
\underline{x_{\nu}(\beta)}=\left\{\left\langle p,\left(\check{p} \uparrow \operatorname{Col}\left(\alpha_{\nu}(p), \alpha_{\nu+1}(p)\right)\right) \uparrow\left(\alpha_{\nu}(p), \beta\right)\right\rangle \mid p \in D(\nu)\right\},
$$

where for $q \in \operatorname{Col}(\gamma, \delta)$

$$
q \uparrow(\gamma, \xi)= \begin{cases}q \uparrow \operatorname{Col}(\gamma, \xi) & \text { if } \gamma \leqslant \xi<\delta \\ \varnothing & \text { otherwise. }\end{cases}
$$

Case 3. $\nu$ is nonlimit and $\nu \notin A$. Let $\nu=\nu^{\prime}+n$ for a limit ordinal $\nu^{\prime}$ and $n \in \omega-\{0\}$.

Subcase 3.1. $n=2 k$ for $k \in \omega-\{0\}$. Let $p=\left\{\left\langle P_{i}, u_{i}, B_{i}\right\rangle,\left\langle M_{<\kappa^{+}}, B\right\rangle \mid i<n\right\}$ $\in D(\nu) \uparrow \mathbf{R}={ }_{\mathrm{df}}\{q \uparrow \mathbf{R} \mid q \in D(\nu)\}$. Then for some $m_{1}<m_{2}<n$,

$$
P_{m_{1}} \cap \kappa=\alpha_{\nu-2}(p) \text { and } P_{m_{2}} \cap \kappa=\alpha_{\nu}(p) .
$$

Notice that the length of $u_{m_{2}}$ must be equal to one. Let us denote by $p \uparrow\left(\alpha_{\nu}(p), \beta\right)$ the set $\left\langle C_{P_{m_{2}}}^{\prime \prime}\left(P_{i}\right) \cap \beta \mid m_{1}<i \leqslant m_{2}\right\rangle$ if $\alpha_{\nu}(p) \leqslant \beta<\alpha_{\nu+1}(p)$, or the empty set otherwise.

We set

$$
\underline{x_{\nu}(\beta)}=\left\{\left\langle p,(\check{p} \uparrow \mathbf{R}) \uparrow\left(\alpha_{\nu}(p), \beta\right)\right\rangle \mid p \in D(\nu)\right\} .
$$

Subcase 3.2. $n=2 k+1$ for $k \in \omega$. Let $q=\left\langle Q_{1}, \ldots, Q_{n}, B\right\rangle \in \mathscr{P}(\gamma, \delta)$. We denote

$$
q \uparrow(\gamma, \xi)= \begin{cases}\left\langle Q_{1} \cap \xi, \ldots, Q_{n} \cap \xi\right\rangle & \text { if } \gamma \leqslant \xi<\delta \\ \varnothing & \text { otherwise }\end{cases}
$$


Define

$$
\underline{x_{\nu}(\beta)}=\left\{\left\langle p,\left(\check{p} \uparrow \mathscr{P}\left(\alpha_{\nu}(p), \alpha_{\nu+1}(p)\right)\right) \uparrow\left(\alpha_{\nu}(p), \beta\right)\right\rangle \mid p \in D(\nu)\right\} .
$$

It completes the definition of $\left\langle x_{\nu}(\beta)\right| \nu<\kappa, \beta$ is a cardinal less than $\left.\kappa\right\rangle$.

LemmA 3.1.2. Let $\nu, \beta<\kappa, \beta$ is a cardinal. Suppose that $p \in D(\nu) \cap G$ and $\alpha_{\nu}(p) \leqslant \beta<\alpha_{\nu+1}(p)$. Then $\bigcup\left(i_{G}\left(x_{\nu}(\beta)\right)\right)=x_{\nu}(\beta)$.

The lemma follows from the definition of $x_{\nu}(\beta)$.

We are now ready to define the main set $\overline{A(G) \text {. }}$

Definition 3.1.3. Let $\mathscr{G}$ be the group of automorphisms defined in $\$ 2$. Set $A(G)=\left\{\pi\left(x_{\nu}(\beta)\right) \mid \pi \in \mathscr{G}, 0<\nu<\kappa, \beta\right.$ is a cardinal less than $\left.\kappa\right\}$ and $A(G)=$ $\left.\overline{i_{G}(A(G))}=\overline{\left\{i_{G}(\pi\right.}\left(x_{\nu}(\beta)\right)\right) \mid \pi \in \mathscr{G}, 0<\nu<\kappa, \beta$ is a cardinal less than $\left.\kappa\right\}$.

3.2. Cardinals in $\overline{\mathrm{H} M \mathrm{D}}(A(G))$. A set $X \in M[G]$ is $M$-definable over $B$. We shall write $X \in M \mathrm{D}(B)$ iff there is a formula $\varphi$ such that

$$
X=\left\{u \mid M[G] \vDash \varphi\left(u, x_{1}, \ldots, x_{n}, B,\left\langle y_{1}, \ldots, y_{k}\right\rangle\right)\right\}
$$

for some $x_{1}, \ldots, x_{n} \in M$ and $y_{1}, \ldots, y_{\kappa} \in B$.

LEMMA 3.2.1 Let $\alpha_{\nu} \leqslant \beta<\alpha_{\nu+1}$ for some $\nu<\kappa$. Then the cardinality of $\beta$ is the same as the cardinality of $\alpha_{\nu}$ in $\mathrm{HM} \mathrm{D}(A(G))$.

Proof. W.l.o.g. let $\beta$ be a cardinal in $M$. If $\nu=0$ or $\nu \in A$, then $i_{G}\left(x_{\nu}\left(\beta^{+}\right)\right) \in$ $A(G)$. So $\cup i_{G}\left(x_{\nu}\left(\beta^{+}\right)\right)=x_{\nu}\left(\beta^{+}\right)=\operatorname{Col}\left(\alpha_{\nu}, \beta^{+}\right) \in \mathrm{H} M \mathrm{D}(A(G))$. Hence $\beta$ is collapsed to $\alpha_{\nu}$ in $\left.\overline{\mathrm{HMD}(A}(G)\right)$.

Let us assume now that $\nu \notin A \cup\{0\}$. Then $\cup i_{G}\left(x_{\nu}(\beta)\right)=x_{\nu}(\beta)$ is the Prikry or the Radin sequence to $\beta$. Let us concentrate on the case when it is the Radin sequence. The proof for the Prikry sequence is really included in this case. We can refer also to [6, Lemma A.2] for the Prikry case. The claim below is its analog for Radin forcing and uses the same idea. So let $\left\langle P_{\tau} \cap \beta \mid \tau<\xi\right\rangle$ be the supercompact Radin sequence restricted to $\beta$, or its large enough subsequence used in $x_{\nu}(\beta)$, where $\xi<\alpha_{\nu}$ is its order type. The lemma follows from the following

Claim. In $M\left[\left\langle P_{\tau} \cap \beta \mid \tau<\xi\right\rangle\right]$ for every $\gamma, \alpha_{\nu} \leqslant \gamma \leqslant \beta$, there is a sequence of sets $\left\langle B_{\tau}^{\gamma} \mid \tau<\xi\right\rangle$ such that:

(1) $\left|B_{\tau}^{\gamma}\right|<\alpha_{\nu}$,

(2) $\bigcup\left\{B_{\tau}^{\gamma} \mid \tau<\xi\right\}=\gamma$,

(3) $\left\langle B_{\tau}^{\gamma} \mid \tau<\xi\right\rangle$ is an increasing sequence, and

(4) $B_{\tau}^{\gamma}$ can be defined over $M$ using only $\left\langle P_{\tau^{\prime}} \cap \beta \mid \tau^{\prime} \leqslant \tau\right\rangle$.

Proof. We shall define such sets by induction on $\gamma$. Set $B_{\tau}^{\alpha_{\nu}}=P_{\tau} \cap \alpha_{\nu}$. Suppose $\left\langle B_{\tau}^{\gamma^{\prime}} \mid \tau<\xi\right\rangle$ is defined for every $\gamma^{\prime}<\gamma$. Let us define $\left\langle B_{\tau}^{\gamma} \mid \tau<\xi\right\rangle$.

Case $1 .|\gamma|^{M}=\alpha$ for $\alpha<\gamma$. Let $f \in M$ be a one-to-one function from $\alpha$ onto $\gamma$. Set $B_{\tau}^{\gamma}=f^{\prime \prime}\left(B_{\tau}^{\alpha}\right)$.

Case 2. $\operatorname{cf}^{M} \gamma \geqslant \alpha_{\nu}$. Set $B_{\tau}^{\gamma}=\bigcup\left\{B_{\tau}^{\bigcup\left(P_{\tau}, \cap \gamma\right)} \mid \tau^{\prime} \leqslant \tau\right\}$.

Case 3. $\operatorname{cf}^{M} \gamma<\alpha_{\nu}$. Let $\left\langle\gamma_{\nu} \mid \delta<\delta_{0}<\alpha_{\nu}\right\rangle \in M$ be a cofinal sequence to $\gamma$. Define

$$
B_{\tau}^{\gamma}=\bigcup\left\{B_{\tau}^{\gamma_{\delta}} \mid \delta<\delta_{0}\right\} \text {. }
$$


The cardinality of $B_{\tau}^{\gamma}$ in $M\left[\left\langle P_{\eta} \cap \beta \mid \eta<\xi\right\rangle\right]$ is less that $\alpha_{\nu}$ since $\left\langle B_{\tau}^{\gamma_{\delta}} \mid \delta<\delta_{0}\right\rangle \in$ $M\left[\left\langle P_{\eta} \cap \beta \mid \eta \leqslant \tau\right\rangle\right]$ and $\alpha_{\nu}$ is a regular cardinal in this model of the claim.

Lemma 3.2.2. Let $\nu<\kappa$ be an ordinal. Suppose $\nu \notin A \cup\{0\}$. Then $\alpha_{\nu}$ is singular in $\mathrm{H} M \mathrm{D}(A(G))$.

Proof. In this case $x_{\nu}\left(\alpha_{\nu}\right)$ is the Radin or Prikry sequence to $\alpha_{\nu}$.

The statement of the lemma holds clearly if $x_{\nu}\left(\alpha_{\nu}\right)$ is the Prikry sequence or it is the Radin sequence of order type less than $\alpha_{\nu}$. Let us assume that $x_{\nu}\left(\alpha_{\nu}\right)=\stackrel{R}{R}\left(\alpha_{\nu}, \alpha_{\nu}\right)$ is the Radin sequence to $\alpha_{\nu}$ of order type $\alpha_{\nu}$. Since $R$, the supercompact Radin forcing, was of the length $\kappa^{+}$, by [4] every limit point $\alpha$ of the Radin sequence $R_{\text {so }}$ will be singular in $M[\stackrel{\circ}{R}]$, since it has a sequence measure of length $<\alpha^{+}$. In our case we know only the sequence of ordinals $R_{s o} \cap \alpha_{\nu}$ and we have no information about measures, but it is really enough. By [14] it is possible to reconstruct in $M\left[R_{s o} \cap \alpha_{\nu}\right]$ a Radin forcing on $\alpha_{\nu}$ of a length less than or equal to the length of the Radin forcing on $\alpha_{\nu}$ which is being used. Hence $\alpha_{\nu}$ is singular in $M\left[R_{s o} \cap \alpha_{\nu}\right]$. However, the reconstructed forcing turns out to be the same forcing that we are using. Since the length of the last one is $\left\langle\alpha_{\nu}^{+}\right.$, it has no repeat points.

Now we would like to show that every $\alpha_{\nu}$ is a cardinal in $\operatorname{HMD}(A(G))$ and that, for $\nu \in A \cup\{0\}, \alpha_{\nu}^{+}$is regular. In order to do this, we first shall show how to shrink the set of conditions to determine elements of $\operatorname{HMD}(A(G))$.

Suppose

$$
\varphi\left(\pi_{1} \underline{x_{\nu_{1}}\left(\beta_{1}\right)}, \ldots, \pi_{n} \underline{x_{\nu_{n}}\left(\beta_{n}\right)}, \underline{A(G)}\right)
$$

is a formula which may also contain some parameters from $M$. Let $p \in D\left(\nu_{1}\right) \cap$ $\cdots \cap D\left(\nu_{n}\right) \cap G$, i.e., $p$ already knows $\alpha_{\nu_{1}}, \ldots, \alpha_{\nu_{n}}$. Now $\mathbf{P}$ is the direct product of $\mathbf{R}$, $\operatorname{Col}(\alpha, \beta)$ and $\mathscr{P}(\gamma, \delta)$ for lot of ordinals $\alpha, \beta, \gamma, \delta$. So $p$ may contain some irrelevant information. Let us shrink it to a condition containing the information only from the forcing notions needed to determine $\mathrm{x}_{\nu_{1}}\left(\beta_{1}\right), \ldots, x_{\nu_{n}}\left(\beta_{n}\right)$.

W.l.o.g. we assume that parameters $\pi_{1} x_{\nu_{1}}\left(\beta_{1}\right), \ldots, \pi_{n} x_{\nu_{n}}\left(\beta_{n}\right)$ in $\varphi$ are ordered in such a way that the following holds for some $0=m_{0} \overline{<m_{1}<m_{2}}<m_{3}<m_{4}=n$ : $i \leqslant i^{\prime}$ implies $\nu_{i} \leqslant \nu_{i^{\prime}}$ and, in case $\nu_{i}=\nu_{i^{\prime}}, \beta_{i} \leqslant \beta_{i^{\prime}}$ for every $i, i^{\prime}, j$ s.t. $j \in\{1,2,3,4\}$ and $i, i^{\prime} \in\left(m_{j-1}, m_{j}\right]$.

Also:

1. If $1 \leqslant i \leqslant m_{1}$, then

$$
\underline{x_{i}}\left(\beta_{i}\right) \text { is the name of } \operatorname{Col}\left(\alpha_{\nu_{i}}, \beta_{i}\right) .
$$

2. If $m_{1}<i \leqslant m_{2}$, then

$$
\underline{x_{\nu_{i}}}\left(\beta_{i}\right) \text { is the name of } \mathscr{P}\left(\alpha_{\nu_{i}}, \beta_{i}\right) .
$$

3. If $m_{2}<i \leqslant m_{3}$, then

$$
\nu_{i} \text { is nonlimit and } x_{\nu_{i}}\left(\beta_{i}\right) \text { is the name of } \stackrel{\circ}{R}\left(\alpha_{\nu_{i}}, \beta_{i}\right) \text {. }
$$

4. If $m_{3}<i \leqslant m_{4}$, then

$$
\nu_{i} \text { is a limit ordinal and } \underline{x_{\nu_{i}}\left(\beta_{i}\right)} \text { is the name of } \stackrel{R}{R}\left(\alpha_{\nu_{i}}, \beta_{i}\right) \text {. }
$$


For $q \in \mathbf{P}$ stronger than $p$ and $i, j \in \omega, 1 \leqslant i \leqslant n=m_{4}, 1 \leqslant j \leqslant 4$, we shall denote by $q(j, i)$ the restriction of $q$ to $\operatorname{Col}\left(\alpha_{\nu_{i}}, \alpha_{\nu_{i}+1}\right)$ if $j=1,0<i \leqslant m_{1}$, or to $\mathscr{P}\left(\alpha_{\nu_{i}}, \alpha_{\nu_{i}+1}\right)$ if $j=2, m_{1}<i \leqslant m_{2}$, or to $\mathbf{R}$ if $j \in\{3,4\}, m_{2}<i \leqslant m_{4}$. We also denote by $\tilde{\beta}_{i}$ the cardinal $\beta_{i}^{\prime}$ for $1 \leqslant j \leqslant 4$ and $i \in\left(m_{j-1}, m_{j}\right]$, where $i^{\prime}, m_{j} \geqslant i^{\prime} \geqslant i$, is the maximal s.t. $\nu_{i^{\prime}}=\nu_{i}$. Clearly, $\tilde{\beta}_{i}=\max \left\{\beta_{i^{\prime}} \mid m_{j} \geqslant i^{\prime} \geqslant i, \nu_{i^{\prime}}=\nu_{i}\right\}$.

LEMMA 3.2.3. For every $q \geqslant p$

$$
q \Vdash \varphi\left(\pi_{1} \underline{x_{\nu_{1}}\left(\beta_{1}\right)}, \ldots, \pi_{n} \underline{x_{\nu_{n}}\left(\beta_{n}\right)}, \underline{A(G)}\right)
$$

(or its negation) iff

$$
\left\langle q(j, i) \mid 1 \leqslant j \leqslant 4, m_{i-1}<i \leqslant m_{j}\right\rangle \Vdash \varphi\left(\pi_{1} \underline{x_{\nu_{1}}\left(\beta_{1}\right)}, \ldots, \pi_{n} \underline{x_{\nu_{n}}\left(\beta_{n}\right)}, \underline{A(G)}\right)
$$

(or its negation).

Proof. It is obvious since if $q \Vdash \varphi$ and some condition stronger than $\langle q(j, i)|$ $\left.1 \leqslant j \leqslant 4, m_{j-1}<i \leqslant m_{j}\right\rangle$ forces $\neg \varphi$, then there are $q_{1}, q_{2} \in \mathbf{P}$ and $\pi \in \mathscr{G}$ such that

(a) $\pi\left(q_{1}\right)=q_{2}$,

(b) $\pi$ is identity on $\mathbf{R}, \operatorname{Col}\left(\alpha_{\nu_{i}}, \alpha_{\nu_{i}+1}\right)\left(1 \leqslant i \leqslant m_{1}\right), \mathscr{P}\left(\alpha_{\nu_{i}}, \alpha_{\nu_{i}+1}\right)\left(m_{1}<i \leqslant m_{2}\right)$,

(c) $q_{1} \Vdash \varphi$ and $q_{2} \Vdash \neg \varphi$.

But it is impossible since such $\pi$ preserves all the names

$$
\pi_{1} \underline{x_{\nu_{1}}\left(\beta_{1}\right)}, \ldots, \pi_{n} \underline{x_{\nu_{n}}\left(\beta_{n}\right)}, \underline{A(G)} .
$$

The proof of the next lemma is standard and based on the homogeneity of the Levy collapse.

LEMMA 3.2.4 For every $q \geqslant p$

$$
q \Vdash \varphi\left(\pi_{1} \underline{x_{\nu_{1}}\left(\beta_{1}\right)}, \ldots, \pi_{n} \underline{x_{\nu_{n}}\left(\beta_{n}\right)}, \underline{A(G)}\right)
$$

or its negation iff

$$
\left\langle q(1, i) \uparrow\left(\alpha_{\nu_{i}}, \tilde{\beta}_{i}\right) \mid 0<i \leqslant m_{1}\right\rangle \sim\left\langle q(j, i) \mid 2 \leqslant j \leqslant 4, m_{j-1}<i \leqslant m_{j}\right\rangle
$$

forces the same.

Now we shall shrink the information from the supercompact Prikry and Radin forcings. Let us first explain the difference here from the Levy collapse. For simplicity we deal with the Prikry case. Let $\beta \in\left[\alpha_{\nu}, \alpha_{\nu+1}\right)$. Every automorphism $\pi \in \mathscr{G}_{2}$ of $\mathscr{P}\left(\alpha_{\nu}, \alpha_{\nu+1}\right)$, s.t. for $P \in \mathscr{P}_{\alpha_{\nu}}\left(\alpha_{\nu+1}\right), \pi(P) \cap \beta=P \cap \beta$, will preserve the name $x_{\nu}(\beta)$. But if we have the name $\rho x_{\nu}(\beta)$ for some automorphism $\rho$, then $\pi \rho x_{\nu}\left(\overline{\beta)=\rho} x_{\nu}(\beta)\right.$ will not always be true. It cannot happen in the Levy collapse case, since automorphisms there were defined coordinatewise.

Let us start with the Prikry case. W.l.o.g. we can assume that $\pi_{i} \in \mathscr{G}_{2}\left(\alpha_{\nu_{i}}, \alpha_{\nu_{i}+1}\right)$ for every $i, m_{1}<i \leqslant m_{2}$. Fix some presentation of $\pi_{i}\left(m_{1}<i \leqslant m_{2}\right)$ as

$$
\pi_{p_{t l_{i}}, q_{i i_{i}}} \circ \cdots \circ \pi_{p_{i 1}, q_{i 1}}
$$


where $p_{i j}, q_{i j}$ are similar conditions in $\mathscr{P}\left(\alpha_{\nu_{i}}, \alpha_{\nu_{i}+1}\right)$ (see $\$ 2$ for definitions). We extend $p$ to a condition $p^{\prime}$ in $G$ s.t.

(1) $p(2, i)=p\left\lceil\mathscr{P}\left(\alpha_{\nu_{i}}, \alpha_{\nu_{i}+1}\right)\right.$ may be different from $p^{\prime}(2, i)=p^{\prime} \uparrow \mathscr{P}\left(\alpha_{\nu_{i}}, \alpha_{\nu_{i}+1}\right)$ only for $m_{1}<i \leqslant m_{2}$,

(2) for every $i, j, m_{1}<i \leqslant m_{2}, j \leqslant l_{i}$, the length of the Prikry sequence in $p^{\prime}(2, i)$ is greater than or equal to the length of the Prikry sequence in $p_{i j}$, and the set of measure one in $p^{\prime}(2, i)$ is contained in the set of measure one in $p_{i j}$.

Notice that (2) implies that $p^{\prime}$ is either incompatible with both $p_{i j}, q_{i j}$, for every $i, j, m_{1}<i \leqslant m_{2}, j \leqslant l_{i}$, or is stronger than one of them.

For simplification of the notation let us denote such $p^{\prime}$ by $p$.

LEMMA 3.2.5. Suppose $q_{1}, q_{2} \geqslant p$ satisfy the following conditions:

(a) $q_{1}(j, i)=q_{2}(j, i)$, for every $j \in\{1,3,4\}$ and $i \in\left(m_{j-1}, m_{j}\right]$,

(b) $q_{1}(2, i)$ and $q_{2}(2, i)$ are similar conditions in $\mathscr{P}\left(\alpha_{\nu_{i}}, \alpha_{\nu_{i}+1}\right)$ and $q_{1}(2, i) \uparrow$ $\left(\alpha_{\nu_{i}}, \beta_{i}\right)=q_{2}(2, i) \uparrow\left(\alpha_{\nu_{i}}, \beta_{i}\right)$ for every $i \in\left(m_{1}, m_{2}\right]$. Then

$$
q_{1} \Vdash \varphi\left(\pi_{1} \underline{x_{\nu_{1}}\left(\beta_{1}\right)}, \ldots, \pi_{n} \underline{x_{\nu_{n}}\left(\beta_{n}\right)}, \underline{A(G)}\right)
$$

iff

$$
q_{2} \Vdash \varphi\left(\pi_{1} \underline{x_{\nu_{1}}\left(\beta_{1}\right)}, \ldots, \pi_{n} \underline{x_{\nu_{n}}\left(\beta_{n}\right)}, \underline{A(G)}\right) .
$$

Proof. It is enough to show that for every $i \in\left(m_{1}, m_{2}\right]$

$$
p \Vdash " \pi_{q_{1}(2, i), q_{2}(2, i)}\left(\pi_{i} x_{\nu_{i}}\left(\beta_{i}\right)\right)=\pi_{i} x_{\nu_{i}}\left(\beta_{i}\right) \text { ". }
$$

Then $\pi=\pi_{q_{1}\left(2, m_{1}+1\right), q_{2}\left(2, m_{1}+1\right)} \circ \cdots \circ \pi_{q_{1}\left(2, m_{2}\right), q_{2}\left(2, m_{2}\right)}$ will take $q_{1}$ to $q_{2}$ and $p$ will force " $\pi \pi_{i} x_{\nu_{i}}\left(\beta_{i}\right)=\pi_{i} x_{\nu_{i}}\left(\beta_{i}\right)$ " for every $i, 1 \leqslant i \leqslant n$.

Let us fix some $i \in \overline{\left(m_{1}, m_{2}\right.}$ ]. We denote $p(2, i), q_{1}(2, i), q_{2}(2, i)$ by $\bar{p}, \overline{q_{1}}, \overline{q_{2}}$ and $\pi \overline{q_{1}}, q_{2}$ by $\pi$. It is enough to show that

$$
\pi_{i} \bar{p} \Vdash \pi_{i}^{-1} \circ \pi \circ \pi_{i}\left(\underline{x_{\nu_{i}}\left(\beta_{i}\right)}\right)=\underline{x_{\nu_{i}}\left(\beta_{i}\right)} .
$$

Set $D=\left\{r \in \mathscr{P}\left(\alpha_{\nu_{i}}, \alpha_{\nu_{i}+1}\right) \mid r \geqslant \pi_{i}(\bar{p})\right.$, the length of the Prikry sequence in $r$ is greater than in $\overline{q_{1}}$ and the set of measure one of $r$ is contained in that of $\left.\overline{q_{1}}\right\}$. Such $D$ is clearly a dense open set above $\pi_{i}(\bar{p})$. Also, by the definition of automorphisms for $\mathscr{P}\left(\alpha_{\nu_{i}}, \alpha_{\nu_{i}+1}\right), \pi \uparrow D: D \rightarrow \mathscr{P}\left(\alpha_{\nu_{i}}, \alpha_{\nu_{i}+1}\right)$. By the choice of $p$, the same is true for $\pi_{p_{i j}, q_{i j}}\left(1 \leqslant j \leqslant l_{i}\right)$. So $\pi_{i}^{-1} \circ \pi \circ \pi_{i} \uparrow D: D \rightarrow \mathscr{P}\left(\alpha_{\nu_{i}}, \alpha_{\nu_{i}+1}\right)$.

Claim. For every $r \in D$

$$
\pi_{i}^{-1} \circ \pi \circ \pi_{i}(r) \uparrow\left(\alpha_{\nu_{i}}, \beta_{i}\right)=r \uparrow\left(\alpha_{\nu_{i}}, \beta_{i}\right) .
$$

Let us first show how the lemma follows from the Claim.

$$
\begin{gathered}
\text { Let } \underline{x}=\left\{\left\langle\pi_{i}^{-1} \circ \pi \circ \pi_{i}(r), r \check{r}\left(\alpha_{\nu_{i}}, \beta_{i}\right)\right\rangle \mid \pi_{i}^{-1} \circ \pi \circ \pi_{i}(r) \in D\right\} \text {. Then } \\
\pi_{i} \bar{p} \Vdash \pi_{i}^{-1} \circ \pi \circ \pi_{i}\left(\underline{x_{\nu_{i}}\left(\beta_{i}\right)}\right)=\underline{x} .
\end{gathered}
$$

For every $r \in D, r^{\prime}=\pi_{i}^{-1} \circ \pi \circ \pi_{i}(r) \in \mathscr{P}\left(\alpha_{\nu_{i}}, \alpha_{\nu_{i}+1}\right)$ and $\pi_{i}^{-1} \circ \pi \circ \pi_{i}\left(r^{\prime}\right)=r$. Hence $\underline{x}=\left\{\left\langle r,\left(\pi_{i}^{-1} \circ \pi \circ \pi_{i}(r)\right) \check{\uparrow}\left(\alpha_{\nu_{i}}, \beta_{i}\right)\right\rangle \mid r \in D\right\}$. Applying the Claim, we obtain that

$$
\underline{x}=\left\{\left\langle r, r \grave{i}\left(\alpha_{\nu_{i}}, \beta_{i}\right)\right\rangle \mid r \in D\right\} .
$$


Then, clearly,

$$
\pi_{i} \bar{p} \Vdash \underline{x_{\nu_{i}}\left(\beta_{i}\right)}=\underline{x} .
$$

Proof. Let

$$
\begin{aligned}
\bar{p} & =\left\langle P_{1}, \ldots, P_{N}, B\right\rangle, \\
\overline{q_{1}} & =\left\langle P_{1}, \ldots, P_{N}, Q_{11}, \ldots, Q_{1 s}, C\right\rangle, \\
\overline{q_{2}} & =\left\langle P_{1}, \ldots, P_{N}, Q_{21}, \ldots, Q_{2 s}, C\right\rangle .
\end{aligned}
$$

By condition (b) in the statement of the lemma,

$$
Q_{1 j} \cap \beta_{i}=Q_{2 j} \cap \beta_{i} \text { for every } j, 1 \leqslant j \leqslant s .
$$

Let $r \in D$ and $r^{\prime}=\pi_{i}^{-1} \circ \pi \circ \pi_{i}(r)$. Set $r(0)=r$. For every $j, l_{j} \geqslant j \geqslant 1$, set

$$
r(j)=\pi_{p_{i,}, q_{i j}} \circ \cdots \circ \pi_{p_{i 1}, q_{i 1}}(r),
$$

i.e., $r(j)=\pi n_{p_{i,}, q_{i}}(r(j-1))$. Let $r^{\prime}\left(l_{i}\right)=\pi\left(r\left(l_{i}\right)\right)$. For every $j, l_{i}>j \geqslant 0$, set

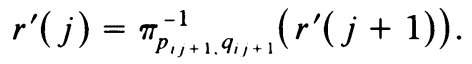

Then $r^{\prime}=r^{\prime}(0)$.

Notice that, since $r \geqslant \pi_{i}(\bar{p})$ and, by the choice of $p, \pi_{i}$ cannot change anything in $p$ above $\left\langle P_{1}, \ldots, P_{N}\right\rangle$, all the elements in the Prikry sequence of $r$ with index greater than $N$ belong to $B$. Also the set of measure one in $r$ is contained in $B$. The same holds for $r(j)\left(1 \leqslant j \leqslant l_{i}\right)$.

Let us prove by induction on $j \in\left[0, l_{i}\right]$ that the following holds:

$$
r(j)=r^{\prime}(j)
$$

or

$$
\begin{aligned}
& r(j)=\left\langle R_{1}, \ldots, R_{N}, Q_{k 1}, \ldots, Q_{k s}, R_{N+1}, \ldots, R_{M}, E\right\rangle, \\
& r^{\prime}(j)=\left\langle R_{1}, \ldots, R_{N}, Q_{k^{\prime} 1}, \ldots, Q_{k^{\prime} s}, R_{N+1}, \ldots, R_{M}, E\right\rangle
\end{aligned}
$$

for some $R_{1}, \ldots, R_{M}, E$ and $k \neq k^{\prime}, k, k^{\prime} \in\{1,2\}$.

The statement $(*)$ for $j=0$ together with (b) implies that $\pi_{i}^{-1} \circ \pi \circ \pi_{i}(r) \uparrow\left(\alpha_{\nu_{i}}, \beta_{i}\right)$ $=r \uparrow\left(\alpha_{\nu}, \beta_{i}\right)$. For $j=l_{i},(*)$ holds by the definition of $\pi$. Suppose it holds for every $j^{\prime}, l_{i} \geqslant j^{\prime}>j$. Let us prove $(*)$ for $j$. By the definitions,

$$
r(j+1)=\pi_{p_{i j+1}, q_{i j+1}}(r(j))
$$

and

$$
\pi_{p_{i j+1}, q_{i j+1}}^{-1}\left(r^{\prime}(j+1)\right)=r^{\prime}(j) .
$$

If $r(j+1)=r^{\prime}(j+1)$, then also $r(j)=r^{\prime}(j)$. Suppose that $r(j+1) \neq r^{\prime}(j+1)$.

Then for $k \neq k^{\prime}, k, k^{\prime} \in\{1,2\}$ and for some $R_{1}, \ldots, R_{M}, E$

$$
r(j+1)=\left\langle R_{1}, \ldots, R_{N}, Q_{k 1}, \ldots, Q_{k s}, R_{N+1}, \ldots, R_{M}, E\right\rangle
$$

and

$$
r^{\prime}(j+1)=\left\langle R_{1}, \ldots, R_{N}, Q_{k^{\prime} 1}, \ldots, Q_{k^{\prime} s}, R_{N+1}, \ldots, R_{M}, E\right\rangle .
$$


By our assumptions on $p$ and the remark above, either $r(j)$ is incompatible with both $p_{i j+1}$ and $q_{i j+1}$ or it is stronger than one of them. Suppose first that $r(j)$ is incompatible with both $p_{i j+1}$ and $q_{i j+1}$. Then $r(j+1)=r(j)$. The Prikry sequence of $p_{i j+1}$ is not an initial segment of $\left\langle R_{1}, \ldots, R_{N}\right\rangle$ or if it is an initial segment, say $\left\langle R_{1}, \ldots, R_{N^{\prime}}\right\rangle$, then some $R_{N^{\prime \prime}}\left(N^{\prime}<N^{\prime \prime} \leqslant N\right)$ does not belong to the set of measure one of $p_{i j+1}$, since otherwise $r(j+1) \geqslant p_{i j+1}$ by the remark above. The same holds for $q_{i j+1}$. But by $(*), r^{\prime}(j+1)$ starts from the same sequence $\left\langle R_{1}, \ldots, R_{N}\right\rangle$. Hence $r^{\prime}(j+1)$ is also incompatible with both $p_{i j+1}$ and $q_{i j+1}$. Then $r^{\prime}(j)=r^{\prime}(j+1)$. So $(*)$ is satisfied.

Now let us assume that $r(j) \geqslant p_{i j+1}$. In case $r(j) \geqslant q_{i j+1}$ the argument is the same. Then $r(j+1) \geqslant q_{i j+1}$. By the choice of $p$ it implies that $\left\langle R_{1}, \ldots, R_{N}, B\right\rangle \geqslant$ $q_{i j+1}$. By the remark above, $E \subseteq B$ and $\left\langle Q_{k 1}, \ldots, Q_{k s}, R_{N+1}, \ldots, R_{M}\right\rangle \in[B]^{s+M-N}$ for $k=1,2$. Then $r^{\prime}(j+1) \geqslant q_{i j+1}$ and, by the definition of $\pi_{p_{i j+1} q_{i j+1}}, r(j)$ and $r^{\prime}(j)$ satisfy $(*)$. This completes the induction.

Now let us consider the case $i \in\left(m_{2}, m_{4}\right]$, i.e., $x_{\nu_{i}}\left(\beta_{i}\right)$ is the name of $\stackrel{\circ}{R}_{(}\left(\alpha_{\nu_{i}}, \beta_{i}\right)$.

W.1.o.g. every $\pi_{i} \in \mathscr{G}_{3}$ for $i \in\left(m_{2}, m_{4}\right]$. Let us fix for every $m_{2} \leqslant i<m_{4}$ a presentation $\pi_{p_{i i_{i}, q_{i i_{i}}}} \circ \cdots \circ \pi_{p_{i 1}, q_{i 1}}$ of $\pi_{i}$, where $p_{i j}, q_{i j}$ are similar conditions in $\mathbf{R}$. As in Lemma 3.2.5 we shall first extend $p$. For every $i \in\left(m_{2}, m_{4}\right]$ and $j \in\left[1, l_{i}\right]$, $\langle Q, v\rangle$ appears in $p_{i j}$ with $v \neq \varnothing$. Let us add to $p \uparrow \mathbf{R}$ some $\langle P, u\rangle$ s.t. $P \cap \kappa=Q$ $\cap \kappa$ if it is possible. Let $p(0)$ be such an extension. Now if, for some $i \in\left(m_{2}, m_{4}\right]$ and $j \in\left[1, l_{i}\right],\langle Q, u, B\rangle$ appears in $p_{i j}$ with $u \neq \varnothing$ and some $\langle P, u, C\rangle$ appears in $p(0) \uparrow \mathbf{R}$, then we replace $C$ in $p(0) \uparrow \mathbf{R}$ by $C \cap B$. Let us denote such an extension of $p(0)$ by $p$.

Notice that, for every $i \in\left(m_{2}, m_{4}\right], 1 \leqslant j \leqslant l_{i}, p \uparrow \mathbf{R}$ or any element of $\mathbf{R}$ similar to it is either incompatible with both $p_{i j}, q_{i j}$ or stronger than any one of them.

Let us first consider the case $i \in\left(m_{2}, m_{3}\right]$, i.e., $\nu_{i}$ is a nonlimit ordinal. Here we are almost in the situation of Lemma 3.2.5, since the fragment of $\mathbf{R}$ we really use in this case is isomorphic to $\mathscr{P}\left(\alpha_{\nu_{i}}, \alpha_{\nu_{i}+1}\right)$.

For $\nu<\kappa$, let us denote by $P(\nu)$ the element $P$ that appears in $p \nmid \mathbf{R}$ s.t. $P \cap \kappa=\alpha_{\nu}$, if there is such a $P$.

Since $p \in D\left(\nu_{1}\right) \cap \cdots \cap D\left(\nu_{n}\right)$ for $i \in\left(m_{2}, m_{3}\right], P\left(\nu_{i}-2\right)$ and $P\left(\nu_{i}\right)$ are defined.

LEMMA 3.2.6. Suppose $q_{1}, q_{2} \geqslant p$ satisfy the following conditions:

(a) $q_{1}(j, i)=q_{2}(j, i)$ for every $j \in\{1,2\}$ and $i \in\left(m_{j-1}, m_{j}\right]$,

(b) $q_{1} \uparrow \mathbf{R}$ and $q_{2} \uparrow \mathbf{R}$ are similar,

(c) for every $i \in\left(m_{2}, m_{3}\right]$,

$$
\left(q_{1} \uparrow \mathbf{R}\right) \uparrow\left(\boldsymbol{\alpha}_{\nu_{i}}, \beta_{i}\right)=\left(q_{2} \uparrow \mathbf{R}\right) \uparrow\left(\boldsymbol{\alpha}_{\nu_{i}}, \beta_{i}\right),
$$

(d) $u=\varnothing$ for every $\langle Q, u, B\rangle$ belonging to $q_{1} \uparrow \mathbf{R}-q_{2} \uparrow \mathbf{R}$ or to $q_{2} \uparrow \mathbf{R}-q_{1} \uparrow \mathbf{R}$, and there exists $i \in\left(m_{2}, m_{3}\right]$ s.t. $P\left(\nu_{i-2}\right) \varsubsetneqq Q \subsetneq P\left(\nu_{i}\right)$.

Then

$$
q_{1} \Vdash \varphi\left(\pi_{1} \underline{x_{\nu_{1}}\left(\beta_{1}\right)}, \ldots, \pi_{n} \underline{x_{\nu_{n}}\left(\beta_{n}\right)}, \underline{A(G)}\right)
$$


iff

$$
q_{2} \Vdash \varphi\left(\pi_{1} \underline{x_{\nu_{1}}\left(\beta_{1}\right)}, \ldots, \pi_{n} \underline{x_{\nu_{n}}\left(\beta_{n}\right)}, \underline{A(G)}\right) .
$$

Proof. Let us denote $p \uparrow \mathbf{R}, q_{1} \uparrow \mathbf{R}, q_{2} \uparrow \mathbf{R}$ by $\bar{p}, \overline{q_{1}}, \overline{q_{2}}$. Set $\pi=\pi \frac{q_{1}, q_{2}}{\text {. It is enough }}$ to show that for every $i \in\left(m_{2}, m_{3}\right]$

$$
\pi_{i} \bar{p} \Vdash \pi_{i}^{-1} \circ \pi \circ \pi_{i}\left(\underline{\left.x_{\nu_{i}}\left(\beta_{i}\right)\right)}\right)=\underline{x_{\nu_{i}}\left(\beta_{i}\right)} .
$$

Set $D^{\prime}=\left\{r \in \mathbf{R} \mid r \geqslant \bar{p}\right.$; for every $i \in\left(m_{2}, m_{3}\right]$ the length of the sequence in $r$ between $P\left(\nu_{i-2}\right)$ and $P\left(\nu_{i}\right)$ is greater than this length in $\overline{q_{1}}$ and the set of measure one for $P\left(\nu_{i}\right)$ in $r$ is contained in that for $\overline{q_{1}}$. This set is dense and open above $\bar{p}$. By the definition of automorphisms, $p$ and $D^{\prime}$, and for every $i \in\left(m_{2}, m_{4}\right]$ and $1 \leqslant j \leqslant l_{i}, \pi_{p_{i}, q_{i j}} \uparrow D^{\prime}: D^{\prime} \rightarrow \mathbf{R}, \pi \uparrow D^{\prime}: D^{\prime} \rightarrow \mathbf{R}$ and $\pi_{i}^{-1} \circ \pi \circ \pi_{i} \uparrow D^{\prime}: D^{\prime} \rightarrow \mathbf{R}$. Set $D=\pi_{i}^{\prime \prime}\left(D^{\prime}\right)$. Then the above is true for $D$.

As in Lemma 3.2.5, the lemma follows from the following

Claim. For every $i \in\left(m_{2}, m_{4}\right], r \in D$,

$$
\pi_{i}^{-1} \circ \pi \circ \pi_{i}(r) \uparrow\left(\alpha_{\nu_{i}}, \beta_{i}\right)=r \uparrow\left(\alpha_{\nu_{i}}, \beta_{i}\right) .
$$

Proof. Let $i \in\left(m_{2}, m_{4}\right), r \in D$ and $r^{\prime}=\pi_{i}^{-1} \circ \pi \circ \pi_{i}(r)$. For every $j \leqslant l_{i}$ we define $r(j), r^{\prime}(j)$ as in Lemma 3.2.5.

Now for every $j \leqslant l_{i}$ the following hold:

$$
r(j)=r^{\prime}(j),
$$

or

(1) for every $\langle R, v, T\rangle \in r(j), v \neq \varnothing$ implies $\langle R, v, T\rangle \in r^{\prime}(j)$,

(2) for every $\langle P, u\rangle$ that appears in $\bar{p}$ with $u \neq \varnothing$ there is a $Q$ s.t. $\langle Q, u\rangle$ appears in $r(j)$,

(3) for every $R \in r(j)\left(r^{\prime}(j)\right)$, if there is no $k \in\left(m_{2}, m_{3}\right]$ s.t. $\alpha_{\nu_{k}-2}<R \cap \kappa<\alpha_{\nu_{k}}$, then $R \in r^{\prime}(j)(r(j))$,

(4) for $l \in\left(m_{2}, m_{3}\right]$ let $R\left(\nu_{l}-2\right)$ and $R\left(v_{l}\right)$ (which appear in $\left.r(j)\right)$ be s.t.

$$
R\left(\nu_{l}-2\right) \cap \kappa=\alpha_{\nu_{l}-2} \text { and } R\left(\nu_{l} \cap \kappa\right)=\alpha_{\nu_{l}} .
$$

Then the sequence between $R\left(\nu_{l}-2\right)$ and $R\left(\nu_{l}\right)$ looks like

$$
\left\langle R_{1}, \ldots, R_{N}, C_{R\left(\nu_{l}\right)}^{-1} \circ C_{P\left(\nu_{l}\right)}^{\prime \prime}\left(Q_{k 1}\right), \ldots, C_{R\left(\nu_{l}\right)}^{-1} \circ C_{P\left(\nu_{l}\right)}^{\prime \prime}\left(Q_{k s}\right), R_{N+1}, \ldots, R_{M}\right\rangle
$$

in $r(j)$, and like

$$
\left\langle R_{1}, \ldots, R_{N}, C_{R\left(\nu_{l}\right)}^{-1} \circ C_{P\left(\nu_{l}\right)}^{\prime \prime}\left(Q_{k^{\prime} 1}\right), \ldots, C_{R\left(\nu_{l}\right)}^{-1} \circ C_{P\left(\nu_{l}\right)}^{\prime \prime}\left(Q_{k^{\prime} s}\right), R_{N+1}, \ldots, R_{M}\right\rangle
$$

in $r^{\prime}(j)$ for some

$$
R_{1}, \ldots, R_{M}, Q_{k 1}, \ldots, Q_{k s}, Q_{k^{\prime} 1}, \ldots, Q_{k^{\prime} s} \in \mathscr{P}_{\alpha_{\nu_{l}}}\left(\alpha_{\nu_{l}+1}\right),
$$

$k \neq k^{\prime}, k, k^{\prime} \in\{1,2\}$, such that

$$
\left\langle P_{1}, \ldots, P_{N}\right\rangle,\left\langle P_{1}, \ldots, P_{N}, Q_{k 1}, \ldots, Q_{k s}\right\rangle,\left\langle P_{1}, \ldots, P_{N}, Q_{k^{\prime} 1}, \ldots, Q_{k^{\prime} s}\right\rangle
$$

are the sequences between $P\left(\nu_{l}-2\right)$ and $P\left(\nu_{l}\right)$ in $\bar{p}, \overline{q_{k}}$ and $\overline{q_{k^{\prime}}}$. 
Now in English, (*) says that $r(j)=r^{\prime}(j)$ or they are the same everywhere except the intervals $\alpha_{\nu_{1}-2}, \alpha_{\nu_{1}}$ for $l \in\left(m_{2}, m_{3}\right]$ and there the difference is as between $\overline{q_{1}}$ and $\overline{q_{2}}$. Also the measures in $r(j), r^{\prime}(j)$ are as in $\bar{p}$.

The proof of $(*)$ is based on the argument used in Lemma 3.2.5 except that the notation here is more complicated. We leave it to the reader.

Now applying (*) for $j=0$, we obtain that $r(0)=r=r^{\prime}(0)=r^{\prime}$ or (1), (2), (3) and (4) in (*) hold. Then

$$
\begin{aligned}
C_{R\left(\nu_{i}\right)}^{\prime \prime}\left(C_{R\left(\nu_{i}\right)}^{-1} \circ C_{P\left(\nu_{i}\right)}^{\prime \prime}\left(Q_{k j}\right)\right) \cap \beta_{i} & =C_{P\left(\nu_{i}\right)}^{\prime \prime}\left(Q_{k j}\right) \cap \beta_{i}=C_{P\left(\nu_{i}\right)}^{\prime \prime}\left(Q_{k^{\prime} j}\right) \cap \beta_{i} \\
& =C_{R\left(\nu_{i}\right)}^{\prime \prime}\left(C_{R\left(\nu_{i}\right)}^{-1} \circ C_{P\left(\nu_{i}\right)}^{\prime \prime}\left(Q_{k^{\prime} j}\right)\right) \cap \beta_{i}
\end{aligned}
$$

for every $j, 1 \leqslant j \leqslant s$. The equality in the middle holds by (c) of the statement of the lemma. Hence $r \uparrow\left(\alpha_{\nu_{i}}, \beta_{i}\right)=r^{\prime} \uparrow\left(\alpha_{\nu_{i}}, \beta_{i}\right)$.

Now remains the last case $i \in\left(m_{3}, m_{4}\right]$, i.e., $\nu_{i}$ is a limit ordinal. We have additional difficulty here since $x_{\nu_{i}}\left(\beta_{i}\right)$ is not a name for all limit points in the supercompact Radin sequence $\left(R_{s l}\right)$ intersected with $\beta_{i}$, but only part of it, namely, all $P \cap \beta_{i}$ for $P \in R_{s l}$ s.t. $\beta_{i} \in P$. The automorphisms of $\mathbf{R}$ do not, in general, preserve the property " $\beta_{i} \in P$ ".

Let us fix an enumeration of all pairs $\langle i, \varphi\rangle$ consisting of $i \in\left(m_{2}, m_{4}\right]$, and functions $\varphi$ defined on subsets of $l_{i}+1$ (where $\pi_{i}=\pi_{p_{i l_{i}} q_{i l_{i}}} \circ \cdots \circ \pi_{p_{i 1} q_{i 1}}$ ) s.t., for every $j \in \operatorname{dom} \varphi, \varphi(j) \in\left\{p_{i j}, q_{i j}\right\}$. We shall extend $p$ step-by-step using on a stage $k_{\text {t }}$ the $k$ th element in the enumeration. Suppose that $p=p^{-1} \leqslant p^{0} \leqslant \cdots \leqslant p^{\bar{k}-1}$ are defined. Let $\langle i, \varphi\rangle$ be the $\bar{k}$ th element in the enumeration. Let $s=\operatorname{dom} \varphi \subseteq l_{i}+1$. If $s=\varnothing$, then set $p^{\bar{k}}=p^{\bar{k}-1}$. Let $\beta(i, \varphi)$ be the $\beta_{i}$ th element of $P\left(\nu_{i}\right)$. Let

$$
\left\langle Q_{j}(i, \varphi) \mid j<k(i, \varphi)\right\rangle=\left\langle Q_{j}^{0}(i, \varphi) \mid j<k(i, \varphi)\right\rangle
$$

be the increasing enumeration of all limit points $Q$ that appear in $p^{\bar{k}}$ s.t. $Q \cap \kappa \leqslant \alpha_{\nu_{i}}$ and $\beta(i, \varphi) \in Q$. Set $\beta(j, i, \varphi)=\beta(i, \varphi)$ for every $j<k(i, \varphi)$.

Suppose now that $s \neq \varnothing$. Let $s=\left\{j_{0}, \ldots, j_{l}\right\}$. For notational simplicity, always assume that $\varphi(j)=p_{i j}$. Also for a while let us drop the indexes $\bar{k}, i, j_{r}$ and denote $p^{\bar{k}} \mid \mathbf{R}$ by $p, \nu_{i}$ by $\nu, \beta_{i}$ by $\beta, \alpha_{\nu_{i}}$ by $\alpha_{\nu}, p_{i j_{r}}$ by $p_{r}$ and $q_{i j_{r}}$ by $q_{r}$ for every $r \leqslant l$.

If there is no $r \in \mathbf{R}$ s.t.

(1) $r \geqslant p_{0}$,

(2) for every $j<l, r(j+1)={ }_{\mathrm{df}} \pi_{p_{j} q_{j}} \circ \cdots \circ \pi_{p_{0} q_{0}}(r) \geqslant p_{j+1}$,

(3) $r(l+1)=\pi_{p_{l} q_{l}}(r(l)) \geqslant p$,

then we do not change $p$.

Suppose otherwise. Let $r \in \mathbf{R}$ satisfy the conditions (1)-(3). Set $p(l+1)=p$ and, for $j \leqslant l, p(j)=\pi_{p_{j} q_{j}}^{-1}(p(j+1))$.

LEMMA 3.2.7. (a) $p$ is stronger than $q_{l}$ and, for every $j, 0<j \leqslant l$, $n p(j)$ is stronger than $p_{j}$ and $q_{j-1}$.

(b) $r(j) \geqslant p(j)$ for every $j \leqslant l+1$.

Proof. We shall prove (a) and (b) simultaneously. $p$ is compatible with $q_{l}$ since $r(l+1)$ is stronger than $p$ and $q_{l}$. But then, by the definition of $p, p \geqslant q_{l}$. Hence 
$p(l)=\pi_{p_{l} q_{l}}^{-1}(p) \geqslant p_{l} . p(l) \leqslant r(l)$ since $r(l)=\pi_{p_{l} q_{l}}^{-1}(r(l+1))$ and $\pi_{p_{l} q_{l}}^{-1}$ is order preserving. Now $r(l)=\pi_{p_{l-1} q_{l-1}}(r(l-1))$ and by $(2) r(l-1) \geqslant p_{l-1}$. Hence $r(l) \geqslant q_{l-1}$. So $p(l)$ and $q_{l-1}$ are compatible. The definition of $p$ implies then that $p(l) \geqslant q_{l-1}$. Continuing in such a way we obtain that (a) and (b) hold for every $j$.

Notice that the lemma implies that $p(0)$ satisfies the conditions (1), (2), (3) and that every $r$ which satisfies (1)-(3) is stronger than $p(0)$.

For $q \in D(\nu)$ let us denote by $P(q, \nu)$ the element $P$ that appears in $q$ s.t. $P \cap \kappa=\alpha_{\nu}$. Let $\bar{\beta}$ be the $\beta$ th ordinal in $P(p(0), \nu)$. Notice that every $p(j)$ $(j \leqslant l+1)$ is similar to $p$ and hence it is in $D(\nu)$. Let $\left\langle Q_{j}^{0} \mid j<k\right\rangle$ be the increasing enumeration of all elements $Q$ that appear in $p(0)$ s.t. $Q \subseteq P(p(0), \nu), \bar{\beta} \in Q$ and $Q$ is the limit (i.e. the sequence of measures $u$ for which $\langle Q, u\rangle$ appears in $p(0)$ is not empty). Suppose $\left\langle Q_{j} \mid j<k\right\rangle$ is the corresponding sequence in $p$, i.e. $Q_{j} \cap \kappa=Q_{j}^{0} \cap$ $\kappa$. Let $\beta(j)=C_{Q}^{-1} \circ C_{Q_{j}^{0}}(\bar{\beta})$ for $j<k$. Notice that $\beta(j)$ does not always equal $\beta\left(j^{\prime}\right)$ or does not always belong to $Q_{j-1}$ for $j, j^{\prime}<k$.

LEMMA 3.2.8. For every $j, 0<j<k$, if some $\left\langle Q_{j}, u, B\right\rangle \in p$ and $\left\langle C_{Q_{j}^{\prime \prime}} P, \nu\right\rangle$ belongs to some element in the sequence of sets of measure one, $B$, then $\beta(j) \in P$.

Proof. Since $p$ and $p(0)$ are similar, $\left\langle Q_{j}^{0}, u, B\right\rangle \in p(0)$. Set $P^{\prime}=C_{Q_{j}^{0}}^{-1}\left(C_{Q_{j}}^{\prime \prime}(P)\right)$. Then $\left\langle P^{\prime}, v\right\rangle$ is addable to $p(0)$. Hence $P^{\prime} \supseteq Q_{j-1}^{0}$. So $\bar{\beta} \in P^{\prime}$. But then $\beta(j)=$ $C_{Q_{i}}^{-1} \circ C_{Q_{i}^{0}}(\bar{\beta}) \in P$.

Let us now define the extension of $p$. Let $\left\langle Q_{0}, u_{0}, B_{0}\right\rangle \in p$ for some $\left\langle u_{0}, B_{0}\right\rangle$. We shrink $B_{0}$ to a sequence of sets of measure one, $B_{0}^{\prime}$, s.t. every $\left\langle C_{Q_{0}}^{\prime \prime}(P), v\right\rangle$ belongs to some member of $B_{0}^{\prime}, \beta(0) \in P$. Let $p^{\prime}$ be such an extension of $p$.

Now let us return to the stage $\bar{k}$ and the old notation. Set $k(i, \varphi)=k$, $\left\langle Q_{j}^{0}(i, \varphi)\right| j\langle k(i, \varphi)\rangle=\left\langle Q_{j}^{0} \mid j<k\right\rangle,\left\langle Q_{j}(i, \varphi)\right| j\langle k(i, \varphi)\rangle=\left\langle Q_{j} \mid j<k\right\rangle$, $\langle\beta(j, i, \varphi)| j\langle k(i, \varphi)\rangle=\langle\beta(j) \mid j<k\rangle$ and $p^{\bar{k}} \mid \mathbf{R}=p^{\prime}, p^{\bar{k}} \backslash \mathbf{R}=p^{\bar{k}-1} \backslash \mathbf{R}$.

It completes the inductive definition of extensions of $p$. Let us denote the final extension by the same letter $p$.

Notice that for every $\langle i, \varphi\rangle$ s.t. $\left\langle Q_{j}(i, \varphi) \mid j<k(i, \varphi)\right\rangle$ is defined, $\left\{Q_{j}(i, \varphi) \mid j<\right.$ $k(i, \varphi)\}=\left\{P \mid P\right.$ is a limit point of $p\left\lceil\mathbf{R}\right.$ and $\left.Q_{0}(i, \varphi) \subseteq P \subseteq P\left(p, \nu_{i}\right)\right\}$.

For a limit point $Q$ of $p \uparrow \mathbf{R}$ s.t. $Q \subseteq P\left(p, \nu_{i}\right)$ for some $i \in\left(m_{3}, m_{4}\right]$, let $\beta(Q)=\max \left\{\beta(j, i, \varphi) \mid i \in\left(m_{2}, m_{4}\right], \varphi\right.$ is a partial function on $l_{i}+1$ s.t., for every $r \in \operatorname{dom} \varphi, \varphi(r) \in\left\{p_{i r}, q_{i r}\right\}, j<k(i, \varphi)$ and $\left.Q_{j}(i, \varphi)=Q\right\}$, if $\beta(j, i, \varphi)$ is defined for at least one such pair $\langle i, \varphi\rangle$ or $\beta(Q)=Q \cap \kappa$ otherwise.

Let $\left\langle Q_{j}(p) \mid j<N\right\rangle$ be the increasing enumeration of all limit points $Q$ of $p \uparrow \mathbf{R}$ s.t., for some $i \in\left(m_{3}, m_{4}\right], Q$ is contained in $P\left(p, v_{i}\right)$. Set $Q_{-1}(p)=\varnothing$.

LEMMA 3.2.9. Suppose $q_{1}, q_{2} \geqslant p$ satisfy the following conditions:

(a) $q_{1}(j, i)=q_{2}(j, i)$ for every $j \in\{1,2\}, i \in\left(m_{j-1}, m_{j}\right]$,

(b) $q_{1} \uparrow \mathbf{R}$ and $q_{2} \uparrow \mathbf{R}$ are similar,

(c) for every $i \in\left(m_{2}, m_{3}\right],\left(q_{1} \uparrow \mathbf{R}\right) \uparrow\left(\alpha_{\nu_{i}}, \alpha_{\nu_{i}+1}\right)=\left(q_{2} \uparrow \mathbf{R}\right) \uparrow\left(\boldsymbol{\alpha}_{\nu_{i}}, \boldsymbol{\alpha}_{\nu_{i}+1}\right)$,

(d) for every $j<N$, and all limit points $Q^{1}$ of $q_{1}$ and $Q^{2}$ of $q_{2}$ s.t. $Q^{1} \cap \kappa=Q^{2} \cap \kappa$ and $Q_{j-1}(p) \subseteq Q^{1} \subseteq Q_{j}(p)$,

$$
Q^{1} \cap \beta\left(Q_{j}(p)\right)=Q^{2} \cap \beta\left(Q_{j}(p)\right) .
$$


Then

$$
q_{1} \Vdash \varphi\left(\pi_{1} \underline{x_{\nu_{1}}\left(\beta_{1}\right)}, \ldots, \pi_{n} \underline{x_{\nu_{n}}\left(\beta_{n}\right)}, \underline{A(G)}\right)
$$

iff

$$
q_{2} \Vdash \varphi\left(\pi_{1} x_{\nu_{1}}\left(\beta_{1}\right), \ldots, \pi_{n} x_{\nu_{n}}\left(\beta_{n}\right), \underline{A(G)}\right) .
$$

Proof. Denote $p \uparrow \mathbf{R}, q_{1} \uparrow \mathbf{R}, q_{2} \uparrow \mathbf{R}$ by $\bar{p}, \overline{q_{1}}, \overline{q_{2}}$. Set $\pi=\pi \overline{q_{1}}, \overline{q_{2}}$. As in the previous cases, it is enough to show that for every $i \in\left(m_{2}, m_{4}\right]$

$$
\pi_{i} \bar{p} \Vdash \pi_{i}^{-1} \circ \pi \circ \pi_{1}\left(\underline{x_{\nu_{i}}\left(\beta_{i}\right)}\right)=\underline{x_{\nu_{i}}\left(\beta_{i}\right)} .
$$

Set $D=\left\{r \in \mathbf{R} \mid r \geqslant \pi_{i} p\right.$; for every $\langle Q, v\rangle$ that appears in $\overline{q_{1}}$ some $\langle P, u\rangle$ with $P \cap \kappa=Q \cap \kappa$ appears in $r$, or it is impossible to add any such pair to $r$; if some $\langle Q, u, B\rangle$ and $\langle P, u, C\rangle$ appear in $q_{1}$ and $r$ with $u \neq \varnothing$, then $\left.C \subseteq B\right\}$.

Notice that every $r \in D$ or any element of $\mathbf{R}$ similar to $r$ is either incompatible with both $\overline{q_{1}}, \overline{q_{2}}$ or stronger than one of them. Clearly, $D$ is a dense and open subset of $\mathbf{R}$ above $\pi_{i} \bar{p}$. By the definitions of automorphisms $p$ and $D$ and, for every $i \in\left(m_{2}, m_{4}\right]$ and $1 \leqslant j \leqslant l_{i}$,

$$
\pi_{p_{i j} q_{i j}} \uparrow D: D \rightarrow \mathbf{R}, \pi \uparrow D: D \rightarrow \mathbf{R} \text { and } \pi_{i}^{-1} \circ \pi \circ \pi_{i} \uparrow D: D \rightarrow \mathbf{R} \text {. }
$$

As in the previous cases, it is enough to prove the following

Claim. For every $i \in\left(m_{2}, m_{4}\right]$ and $r \in D$,

$$
\pi_{i}^{-1} \circ \pi \circ \pi_{i}(r) \uparrow\left(\alpha_{\nu_{i}}, \beta_{i}\right)=r \uparrow\left(\alpha_{\nu_{i}}, \beta_{i}\right) .
$$

Proof. Suppose $r$ is in $D$ and $i \in\left(m_{2}, m_{4}\right]$. Let us define by induction a function $\varphi$ on a subset of $l_{i}+1$. If there is no $j, 1 \leqslant j \leqslant l_{i}$, s.t. $r \geqslant p_{i j}$ or $r \geqslant q_{i j}$, then let $\varphi=\varnothing$. Otherwise we shall define simultaneously the domain $\left\{j_{t} \mid t \leqslant k\right\}$ and the range of $\varphi$. Let $j_{0}$ be the least $j, 1 \leqslant j \leqslant l_{i}$, s.t. $r \geqslant p_{i j}$ or $r \geqslant q_{i j}$.

Set

$$
\varphi\left(j_{0}\right)= \begin{cases}p_{i j} & \text { if } r \geqslant p_{i j} \\ q_{i j} & \text { if } r \geqslant q_{i j}\end{cases}
$$

Suppose $\left\langle\left\langle j_{t}, \varphi\left(j_{t}\right)\right\rangle \mid t \leqslant k^{\prime}\right\rangle$ is defined. If there is no $j, k^{\prime}<j \leqslant l_{i}$, s.t. $r_{k^{\prime}+1}=$ $\pi_{p_{i j}, q_{i j k^{\prime}}} \circ \cdots \circ \pi_{p_{i j 0} q_{i j o}}(r)$ is stronger that $p_{i j}$ or $q_{i j}$, then let $k=k^{\prime}$ and $\varphi=$ $\left\{\left\langle j_{t}, \varphi\left(j_{t}\right)\right\rangle \mid t \leqslant k\right\}$. Otherwise let $j_{k^{\prime}+1}$ be the least $j, k^{\prime}<j \leqslant l_{i}$, s.t. $r_{k^{\prime}+1} \geqslant p_{i j}$ or $q_{i j}$.

Set

$$
\varphi\left(j_{k^{\prime}+1}\right)= \begin{cases}p_{i j_{k^{\prime}+1}} & \text { if } r_{k^{\prime}+1} \geqslant p_{i j_{k^{\prime}+1}} \\ q_{i j_{k^{\prime}+1}} & \text { otherwise. }\end{cases}
$$

Now if $r_{k+1}$ is not stronger than $q_{1}$ or $q_{2}$, then $\pi\left(r_{k+1}\right)=r_{k+1}$ and hence $\pi_{i}^{-1} \pi \pi_{i}(r)=r$, where $r_{k+1}=r$ if $\varphi=\varnothing$. Obviously, the statement of the Claim holds in this case.

Suppose that $r_{k+1} \geqslant q_{1}$ (or $q_{2}$ which is the same). Then $r$ satisfies the conditions (1), (2), (3) before Lemma 3.2.7 for the pair $\langle i, \varphi\rangle$. We proceed now as in Lemma 
3.2.7. By the choice of $p$, it is incompatible with both $p_{i l_{i}}$ and $q_{i l_{i}}$ or it is stronger than $p_{i l_{i}}$ or $q_{i l_{i}}$

In the first case, $l_{i} \notin \operatorname{dom} \varphi$; in the second one, we must have $l_{i}=j_{k}$ since $r_{k+1} \geqslant p$. In the same way, for every $j_{k} \leqslant j \leqslant l_{i}, p$ is incompatible with both $p_{i j}$ and $q_{i j}$ and then $j>j_{k}$, or $p$ is stronger than $p_{i j}$ or $q_{i j}$ and then $j=j_{k}$. Hence

$$
p(k)={ }_{\mathrm{df}} \pi_{p_{i j_{k}} q_{i j_{k}}}^{-1}(p)=\pi_{p_{i j_{k}} q_{i j_{k}}}^{-1} \circ \cdots \circ \pi_{p_{i i_{i}}, q_{i i_{i}}}^{-1}(p) .
$$

Applying the same argument to $p(k)$ and $r_{k}=\pi_{p_{i j_{k}} q_{i j_{k}}}^{-1}\left(r_{k+1}\right)$ we obtain

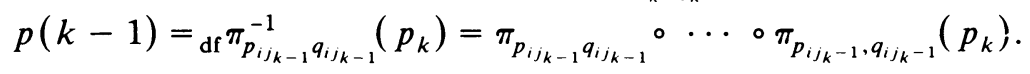

Continuing, we have, as in Lemma 3.2.7,

$$
p(0)={ }_{\mathrm{df}} \pi_{p_{i j 0} q_{i, i_{0}}}^{-1}(p(1))=\pi_{p_{i j 0} q_{i j_{0}}}^{-1} \circ \cdots \circ \pi_{p_{i j k} q_{i j_{k}}}^{-1}(p)=\pi_{p_{i 1} q_{i 1}}^{-1} \circ \cdots \circ \pi_{p_{i i_{i}} q_{i i_{i}}}^{-1}(p) .
$$

Now, since $r_{k+1}, \pi\left(r_{k+1}\right) \geqslant p$, it follows that, for every $t \leqslant k$,

$$
r_{k-t}^{\prime}={ }_{\mathrm{df}} \pi_{p_{i j_{k-1}} q_{i j_{k-t}}}^{-1}\left(r_{k-t+1}^{\prime}\right)=\pi_{p_{i j_{k-t}} q_{i j_{k-t}}}^{-1} \cdots \circ \pi_{p_{i j_{k-t+1}-1} q_{i j_{k-t+1^{-1}}}}^{-1}\left(r_{k+1}^{\prime}\right) \text {, }
$$

where $r_{k+1}^{\prime}=\pi\left(r_{k+1}\right)$. So

$$
r_{0}^{\prime}=\pi_{p_{i j 0} q_{i j_{0}}}^{-1} \circ \cdots \circ \pi_{p_{i j_{k}} q_{i j_{k}}}^{-1}\left(\pi\left(r_{N+1}\right)\right)=\pi_{i}^{-1}\left(\pi\left(\pi_{i}(r)\right)\right) \text {. }
$$

Case 1. $i \in\left(m_{2}, m_{3}\right]$. It is enough to show that, for every $t \leqslant k+1$, any $Q \in \mathscr{P}_{\alpha_{\nu_{i}}}\left(\alpha_{\nu_{i}+1}\right)$ s.t. $P\left(p, \nu_{i}-2\right) \cap \kappa<Q \cap \kappa$, appears in $r_{t}$ iff it appears in $r_{t}^{\prime}$. For $t=k+1$ it follows from the condition (c) of the lemma. Suppose it holds for $t+1$. Let $\varphi\left(j_{t}\right)=p_{i j_{t}}$ (if $\varphi\left(j_{t}\right)=q_{i j_{t}}$ the argument is the same). Then $r_{t} \geqslant p_{i j_{t}}$ and $r_{t+1}=\pi_{p_{i j} q_{i j}}\left(r_{t}\right) \geqslant q_{i j_{t}}$. As in Lemma 3.2.7, then also $r_{t}^{\prime} \geqslant p_{i j_{t}}$ and $r_{t+1}^{\prime}=\pi_{p_{i j_{t}} q_{i j_{t}}}\left(r_{t}^{\prime}\right)$ $\geqslant q_{i j_{i}}$. Now it follows from the definitions of $\pi_{p_{i j} q_{i j}}, p$, and the assumption for $t+1$ that, for every $Q \in \mathscr{P}_{\alpha_{\nu_{i}}}\left(\alpha_{\nu_{i}+1}\right)$ s.t. $P\left(p, \nu_{i}-2\right) \cap \kappa<Q \cap \kappa, Q$ appears in $r_{t}$ iff $Q$ appears in $r_{t}^{\prime}$.

Case 2. $i \in\left(m_{3}, m_{4}\right]$. Let $\bar{\beta}$ be the $\beta_{i}$ th ordinal in $P\left(r, \nu_{i}\right)$. By arguments above, $P\left(r, \nu_{i}\right)=P\left(p(0), \nu_{i}\right)=P\left(r^{\prime}, \nu_{i}\right)$. Suppose $Q$ is a limit point of $r$ s.t. $Q \subseteq P\left(r, \nu_{i}\right)$ and $\bar{\beta} \in Q$. Let $j<k(i, \varphi)$ be the least so that $Q_{j}^{0}(i, \varphi) \supseteq Q$. Then

$$
Q_{j}(i, \varphi) \supseteq Q^{\prime}={ }_{\mathrm{df}} C_{Q_{j}(i, \varphi)}^{-1}{ }^{\circ} C_{Q_{j}^{\prime}(i, \varphi)}^{\prime \prime}(Q) \text { and } \beta(j, i, \varphi)=C_{Q_{j}(i, \varphi)}^{-1} \circ C_{Q_{j}^{0}(i, \varphi)}(\bar{\beta}) \text {. }
$$

Let $Q^{1}$ be the least limit point in $q_{1}$ s.t. $Q^{\prime} \subseteq Q^{1} \subseteq Q_{j}(i, \varphi)$. Let $Q^{2}$ appear in $q_{2}$ s.t. $Q^{2} \cap \kappa=Q^{1} \cap \kappa$. Set $Q^{\prime \prime}=C_{Q^{2}}^{-1} \circ C_{Q^{1}}^{\prime \prime}\left(Q^{\prime}\right)$. Then $Q^{\prime \prime}$ appears in $\pi\left(\pi_{i}(r)\right)=\pi\left(r_{N+1}\right)$ $=r_{N+1}^{\prime}$.

By Lemma 3.2.8, $\beta(j, i, \varphi) \in Q^{\prime \prime}, Q^{\prime} \cap \beta(j, i, \varphi)=Q^{\prime \prime} \cap \beta(j, i, \varphi)$, since $\left.\beta\left(Q_{j}(i, \varphi)\right) \geqslant \beta(j, i, \varphi)\right)$ and, by $(\mathrm{d}), Q^{1} \cap \beta\left(Q_{j}(i, \varphi)\right)=Q^{2} \cap \beta\left(Q_{j}(i, \varphi)\right)$. Let $\tilde{Q}=C_{Q_{j}^{0}(i, \varphi)}^{-1} \circ C_{Q_{j}(i, \varphi)}\left(Q^{\prime \prime}\right)$. Then $\tilde{Q}$ is a limit point of $r_{0}^{\prime}, \bar{\beta} \in \tilde{Q}$ and $\tilde{Q} \cap \bar{\beta}=Q \cap$ $\bar{\beta}$. So we proved that

$$
r \uparrow\left(\alpha_{\nu_{i}}, \beta_{i}\right) \subseteq r_{0}^{\prime} \uparrow\left(\alpha_{\nu_{i}}, \beta_{i}\right) .
$$

The opposite direction is the same.

Let us now combine Lemmas 3.2.3-3.2.6 and 3.2.9. Let $p \in D\left(\nu_{1}\right) \cap \cdots \cap D\left(\nu_{n}\right)$ $\cap G$ be a condition extended as in 3.2.5, 3.2.6 and 3.2.9. 
THEOREM 3.2.10. Suppose $q_{1}, q_{2} \geqslant p$ are such that:

(1) $q_{1} \uparrow \mathbf{R}$ and $q_{2} \uparrow \mathbf{R}$ are similar,

(2) $q_{1}(2, i)$ and $q_{2}(2, i)$ are similar for every $i \in\left(m_{1}, m_{2}\right]$,

(3) $q_{i}(j, i)\left|\left(\alpha_{\nu_{i}}, \beta_{i}\right)=q_{2}(j, i)\right|\left(\alpha_{\nu_{i}}, \beta_{i}\right)$ for every $j \in\{1,2,3\}$ and $i \in\left(m_{j-1}, m_{j}\right]$,

(4) for every $j<N$ and all limit points $Q^{1}$ of $q_{1} \uparrow \mathbf{R}$ and $Q^{2}$ of $q_{2} \uparrow \mathbf{R}$ s.t. $Q^{1} \cap \kappa=Q^{2} \cap \kappa$ and $Q_{j-1}(p) \subseteq Q^{1} \subseteq Q_{j}(p)$,

$$
Q^{1} \cap \beta\left(Q_{j}(p)\right)=Q^{2} \cap \beta\left(Q_{j}(p)\right) \text {. }
$$

Then

$$
q_{1} \Vdash \varphi\left(\pi_{1} \underline{x_{\nu_{1}}\left(\beta_{1}\right)}, \ldots, \pi_{n} \underline{x_{\nu_{n}}\left(\beta_{n}\right)}, \underline{A(G)}\right)
$$

iff

$$
q_{2} \Vdash \varphi\left(\pi_{1} \underline{x_{\nu_{1}}\left(\beta_{1}\right)}, \ldots, \pi_{n} \underline{x_{\nu_{n}}\left(\beta_{n}\right)}, \underline{A(G)}\right) .
$$

The proof follows from Lemmas 3.2.3-3.2.6 and 3.2.9.

REMARK. We can replace $\varphi\left(y_{1}, \ldots, y_{n+1}\right)$ by any other formula. What we used to define $p$ was only the sequence of the names $\left\langle\pi_{1} x_{\nu_{1}}\left(\beta_{1}\right), \ldots, \pi_{n} \underline{x_{\nu_{n}}\left(\beta_{n}\right)}\right\rangle$.

THEOREM 3.2.11. For every $\nu<\kappa$ :

(i) every subset of $\alpha_{\nu}$ in $\mathrm{H} M \mathrm{D}(A(G))$ is in $M\left[G \uparrow \alpha_{\nu}\right]$,

(ii) $\alpha_{\nu}$ is a cardinal in $\mathrm{H} M \mathrm{D}(A(G))$,

(iii) if $\nu+1 \in A$, then $\alpha_{\nu+1}$ is a regular cardinal in $\operatorname{HM} \mathrm{D}(A(G))$.

Proof. Let us start with (i). Let $a \subseteq \alpha_{\nu}$ be in $\operatorname{HMD}(A(G))$. Then $a=\{\alpha<$ $\left.\alpha_{\nu} \mid M[G] \vDash \varphi\left(\alpha, i_{G}\left(\pi_{1} x_{\nu_{1}}\left(\beta_{1}\right)\right), \ldots, i_{G}\left(\pi_{n} x_{\nu_{n}}\left(\beta_{n}\right)\right), A(G)\right)\right\}$ for some formula $\varphi\left(x_{0}, x_{1}, \ldots, x_{n}, x_{n+1}\right)$ which may also contain some parameters from $M$. Let us denote $\varphi\left(x_{0}, \pi_{1} x_{\nu_{1}}\left(\beta_{1}\right), \ldots, \pi_{n} x_{\nu_{n}}\left(\beta_{1}\right), A(G)\right)$ by $\Psi\left(x_{0}\right)$. W.l.o.g. we can assume that $\pi_{1} x_{\nu_{1}}\left(\beta_{1}\right), \ldots, \overline{\pi_{n} x_{\nu_{n}}\left(\beta_{n}\right)}$ are ordered as in Theorem 3.2.10. Let $p \in \mathbf{P} \cap D(\nu+2) \cap$ $\left.\cap \overline{\left\{D\left(\nu_{i}+2\right) \mid 1 \leqslant i \leqslant n\right.}\right\} \cap G$ be as in Theorem 3.2.10. We also preserve all the notation of 3.2.10. The relevant part of $P$ will be the direct product of $\left\{\operatorname{Col}\left(\alpha_{\nu_{i}} \beta_{i}\right) \mid\right.$ $\left.1 \leqslant i \leqslant m_{1}\right\},\left\{\mathscr{P}\left(\alpha_{\nu_{i}}, \beta_{i}\right) \mid m_{1}<i \leqslant m\right\}$ and $\mathbf{R}$. Let us denote this product by $\overline{\mathbf{P}}$. Assume also that $p \in \overline{\mathbf{P}}$ and every $q \in \overline{\mathbf{P}}$ is stronger than $p$. We shall show that already the part of forcing below $\alpha_{\nu}$ determines $a$. The idea of the proof is to combine the closure properties of the Levy collapse, and the supercompact Prikry and the supercompact Radin forcings.

Let us denote $\left\langle q(j, i) \mid j=1,2, \alpha_{\nu_{i}} \leqslant \alpha\right\rangle^{\wedge} q^{\prime}$ by $q \uparrow \alpha$ for $q \in \overline{\mathbf{P}}$ and $\alpha<\kappa$ appearing in $p \uparrow \mathbf{R}$ or equal to $\beta^{*}$, and for some $\beta$ appearing in $p \uparrow \mathbf{R}$, where $q^{\prime}=\{\langle P, u, T\rangle \in q|\mathbf{R}| P \cap \kappa \leqslant \alpha\}$, and denote $\left\langle q(j, i) \mid j=1,2, \alpha_{\nu_{i}}>\alpha\right\rangle q^{\prime}$ by $q \backslash \alpha$, where $q^{\prime}=\{\langle P, u, T\rangle \in q \uparrow \mathbf{R} \mid P \cap \kappa>\alpha\}$. Also let

$$
\overline{\mathbf{P}} \mid \alpha=\{q \uparrow \alpha \mid q \in \overline{\mathbf{P}}\} \text { and } \overline{\mathbf{P}} \backslash \boldsymbol{\alpha}=\{q \backslash \alpha \mid q \in \mathbf{P}\} .
$$

Clearly, for every $q_{1} \in \overline{\mathbf{P}} \uparrow \boldsymbol{\alpha}$ and $q_{2} \in \overline{\mathbf{P}} \backslash \alpha, q_{1}{ }^{\imath} q_{2} \in \overline{\mathbf{P}}$, where $q_{1}{ }^{\imath} q_{2}$ is the condition generated by $q_{1}$ and $q_{2}$ in the obvious way.

Let $q \in \overline{\mathbf{P}}$ and $\vec{C}=\left\langle C_{1}, \ldots, C_{m+1}\right\rangle$ be s.t. $C_{1}, \ldots, C_{m}, C_{m+1}$ are sequences of sets of measure one for sequences of measures $u_{1}, \ldots, u_{n}, M_{<\kappa^{+}}$, where $\left\langle P_{1}, u_{1}, B_{1}\right\rangle, \ldots$, 
$\left\langle P_{m}, u_{m}, B\right\rangle,\left\langle M_{<\kappa^{+}}, B\right\rangle$ appear in $q \uparrow \mathbf{R}$ for some $P_{1}, \ldots, P_{m}, B_{1}, \ldots$, $B_{n}, B$. Denote by $q^{\wedge} \vec{C}$ the condition obtained from $q$ by replacing in $q \uparrow \mathbf{R}$ every $\left\langle P_{j}, u_{j}, B_{j}\right\rangle$ by $\left\langle P_{j}, u_{j}, C_{j} \cap B_{j}\right\rangle$ for $1 \leqslant j \leqslant m$ and $\left\langle M_{<\kappa^{+}}, B\right\rangle$ by $\left\langle M_{<\kappa^{+}}, C_{m+1} \cap\right.$ $B\rangle$. If for every $j, 1 \leqslant j \leqslant m, P_{m} \cap \kappa>\alpha$, then we shall say that $\vec{C}$ is above $\alpha$.

Let $\left\langle\alpha(j) \mid j \leqslant M \leqslant m_{4}\right\rangle$ be the increasing enumeration of all $\alpha_{\nu_{i}}\left(1 \leqslant i \leqslant m_{4}\right)$ s.t. $\nu_{i}>\nu$. Set $\alpha(0)=\alpha_{\nu}$.

We proved Theorem 3.2.10 for the similar conditions $q_{1}, q_{2} \geqslant p$. The notion of similarity is an equivalence relation, but the number of equivalence classes is too large. Let us define a weaker notion with fewer equivalence classes.

Definition 3.2.12. For $q_{1}, q_{2}$ in $\overline{\mathbf{P}}$ we say $q_{1}, q_{2}$ are almost similar and write $q_{1} \approx q_{2}$ iff

(1) for every $q_{1}^{\prime} \geqslant q_{1}$ there is $q_{2}^{\prime} \geqslant q_{2}$, so that, for some direct extension $\bar{q}_{1}^{\prime}$ of $q_{1}^{\prime}$ (i.e. obtained only by shrinking sets of measure one Prikry and Radin parts of $q_{1}^{\prime}$ ), $\bar{q}_{1}^{\prime}$ and $q_{2}^{\prime}$ satisfy conditions (1)-(4) of Theorem 3.2.10,

(2) for every $q_{2}^{\prime} \geqslant q_{2}$ there is $q_{1}^{\prime} \geqslant q_{1}$ so that, for some direct extension $\bar{q}_{2}^{\prime}$ of $q_{2}^{\prime}, q_{1}^{\prime}$ and $\bar{q}_{2}^{\prime}$ satisfy conditions (1)-(4) of Theorem 3.2.10.

It is easy to check that $\approx$ is an equivalence relation on $\overline{\mathbf{P}}$.

LeMma 3.2.13. Let $q_{1}, q_{2}$ be almost similar, $\gamma<\alpha_{\nu}, i \in 2$. Then $q_{1} \Vdash{ }^{i} \Psi(\check{\gamma})$ iff $q_{2} \Vdash{ }^{i} \Psi(\check{\gamma})$, where ${ }^{i} \Psi$ is $\Psi$ if $i=0$ and $\neg \Psi$ if $i=1$.

Proof. Suppose $q_{1} \Vdash \Psi(\check{\gamma})$ and $q_{2} \nVdash \Psi(\check{\gamma})$. Then there is $q_{2}^{\prime} \geqslant q_{2}$ and $q_{2}^{\prime}$ $\Vdash \neg \Psi(\check{\gamma})$. Let $q_{1}^{\prime}$ and $\bar{q}_{2}^{\prime}$ be as in 3.2.12(2). Then $q_{1}^{\prime} \Vdash \Psi(\check{\gamma})$ and $\bar{q}_{2}^{\prime} \Vdash \neg \Psi(\check{\gamma})$. But $q_{1}^{\prime}, \bar{q}_{2}^{\prime}$ satisfy conditions (1)-(4) of Theorem 3.2.10. So it is impossible which forms a contradiction and the lemma is proved.

Definition 3.2.14. Let $\alpha<\kappa$ appear in $p$ or, for some $\beta$, let $\alpha=\beta^{*}$ appear in $p$. For $q_{1}, q_{2} \in \overline{\mathbf{P}} \uparrow \alpha$ we say $q_{1}, q_{2}$ are almost similar $\left(q_{1} \approx q_{2}\right)$ if $q_{1} \uparrow p \backslash \alpha$, and $q_{2} \uparrow p \backslash \alpha$ are almost similar.

Now let us count the number of $\approx$-equivalence classes.

LEMMA 3.2.15. Let $\alpha_{\xi}$ appear in $p$ or $\alpha_{\xi-1}$ appear in $p$ for some $\xi<\kappa$. Then there is $\delta$ so that $p \Vdash \delta<\alpha_{\xi+1}$ and there are less than $\delta \approx$-equivalence classes of elements of $\overline{\mathbf{P}} \uparrow \alpha_{\xi}$.

Proof. First notice that, in case $\alpha_{\xi}$ appears in $p, \alpha_{\xi+1}=\alpha_{\xi}^{*}$, and so $p$ already knows $\alpha_{\xi+1}$. Also if $\alpha_{\xi-1}$ appears in $p$ (i.e. $\alpha_{\xi}=\alpha_{\xi-1}^{*}$ ) and $\alpha_{\xi-1} \in\left\{\alpha_{\nu_{i}} \mid 1 \leqslant i \leqslant m_{4}\right\}$, then $p$ knows $\alpha_{\xi+1}$, since then $p \in D(\xi+1)$.

We consider few cases.

Case 1. $\xi$ is not a limit ordinal and $\alpha_{\xi} \notin\left\{\alpha_{\nu_{1}} \mid 1 \leqslant i \leqslant m_{3}\right\}$. Then set $\delta=\left(2^{2^{\alpha_{\xi}}}\right)^{+}$. Let $\left\{q_{\tau} \mid \tau<\delta\right\}$ be a subset of $\overline{\mathbf{P}} \uparrow \alpha_{\xi}$. We would like to find almost two similar elements in this set. W.l.o.g. we can assume that $q_{\tau_{1}} \uparrow \alpha_{\xi-1}=q_{\tau_{2}} \uparrow \alpha_{\xi-1}$ for every $\tau_{1}, \tau_{2}<\delta$ since the cardinality of $\overline{\mathbf{P}} \uparrow \ngtr a_{\xi-1}$ is less than $\delta$. Now if $\alpha_{\xi}=\alpha^{*}$ for some $\alpha$ in $p$, then $\overline{\mathbf{P}} \uparrow \alpha_{\xi}=\overline{\mathbf{P}} \uparrow \alpha_{\xi-1}$, and hence $q_{\tau_{1}}=q_{\tau_{2}}$ for every $\tau_{1}, \tau_{2}<\delta$. Otherwise $\alpha_{\xi}$ appears in $p$. But since $\xi$ is nonlimit and $\alpha_{\xi} \notin\left\{\alpha_{\nu_{i}} \mid 1 \leqslant i \leqslant m_{3}\right\}$, then $\alpha_{\xi}$ also cannot be in $\left\{\alpha_{\nu_{i}} \mid 1 \leqslant i \leqslant m_{4}\right\}$. (Every $\nu_{i}, m_{3}<i \leqslant m_{4}$, is a limit ordinal.) Then we have no restrictions on the forcing between $\alpha_{\xi}$ and $\alpha_{\xi+1}=\alpha_{\xi}^{*}$ in Theorem 3.2.10. 
Let $\langle P(p, \xi), u\rangle$ appear in $p$. We denote by $R\left(p, \alpha_{\xi}, \alpha_{\xi+1}\right)$ the fragment of $\mathbf{R}$ between $P(p, \xi-2)$ and $P(p, \xi)$, i.e. $R\left(p, \alpha_{\xi}, \alpha_{\xi+1}\right)=\left\{\left\langle Q_{1}, \ldots, Q_{n}, B\right\rangle \mid\right.$ for some $q \in \mathbf{R}, q \geqslant p \uparrow \mathbf{R},\langle P(p, \xi), u, B\rangle \in q$ and $\left\langle Q_{1}, \ldots, Q_{n}\right\rangle$ is the sequence in $q$ between $P(p, \xi-2)$ and $P(p, \xi)\}$.

We shall denote $\left\langle Q_{1}, \ldots, Q_{n}, B\right\rangle$ by $q \mid R\left(p, \alpha_{\xi}, \alpha_{\xi+1}\right)$. The order on $R\left(p, \alpha_{\xi}, \alpha_{\xi+1}\right)$ is defined as for supercompact Prikry forcing using the measure $u$.

Let $\tau_{1}, \tau_{2}<\delta$. It is enough to show that

$$
q_{\tau_{1}}\left|R\left(p, \alpha_{\xi}, \alpha_{\xi+1}\right)=_{\mathrm{df}} q_{1}, q_{\tau_{2}}\right| R\left(p, \alpha_{\xi}, \alpha_{\xi+1}\right)
$$

are almost similar. Work in $R\left(p, \alpha_{\xi}, \alpha_{\xi+1}\right)$. Let $q_{1}^{\prime} \geqslant q_{1}$ and choose $q_{2}^{\prime} \geqslant q_{2}$ with the same length of the sequence as in $q_{1}^{\prime}$ and with the set of measure one contained in those of $q_{1}^{\prime}$. But then a direct extension of $q_{1}^{\prime}$ is similar to $q_{2}^{\prime}$.

The same argument gives converse direction. Hence $q_{1} \approx q_{2}$. So $q_{\tau_{1}} \approx q_{\tau_{2}}$.

Case 2. $\xi$ is not a limit ordinal and $\alpha_{\xi}=\alpha_{\nu_{i}}$ for some $i, 1 \leqslant i \leqslant m_{3}$. Then set $\delta=\left(2^{2^{\beta_{i}}}\right)^{+}$. Let $\left\{q_{\tau} \mid \tau<\delta\right\} \subseteq \overline{\mathbf{P}} \mid \alpha_{\xi}$. The cardinality of $\overline{\mathbf{P}} \mid \alpha_{\xi-1}$ is less than $\delta$. W.l.o.g. we can assume that, for every $\tau_{1}, \tau_{2}<\delta, q_{\tau_{1}} \uparrow \alpha_{\xi-1}=q_{\tau_{2}} \uparrow \alpha_{\xi-1}$. Also we can assume that $q_{\tau_{1}}(j, i) \uparrow\left(\alpha_{\nu_{i}}, \beta_{n_{i}}\right)=q_{\tau_{2}}(j, i) \uparrow\left(\alpha_{\nu_{i}}, \beta_{n_{i}}\right)$, where $j=1$ if $1 \leqslant i \leqslant m_{1}$ and $j=2$ if $m_{1}<i \leqslant m_{2}$, since the total number of such restrictions is less than $\delta$. Now in case $1 \leqslant i \leqslant m_{1}$, we are finished. Then every $q_{\tau}(1, i)$ is a condition in the Levy collapse and it means that $q_{\tau_{1}}, q_{\tau_{2}}$ are almost similar for $\tau_{1}, \tau_{2}<\delta$. In case $m_{1}<i \leqslant m_{2}$, we need to work a little more. In this case $q_{\tau}(2, i)$ is a condition in $\mathscr{P}\left(\alpha_{\nu_{i}}, \alpha_{\nu_{i}+1}\right)$. Let $B_{\tau}$ be the set of measure one that appears in $q_{\tau}(2, i)$ for $\tau<\delta$. Set $\bar{B}_{\tau}=\left\{\left\langle Q_{1} \cap \beta_{i}, \ldots, Q_{n} \cap \beta_{i}\right\rangle \mid n<\omega,\left\langle Q_{1}, \ldots, Q_{n}\right\rangle \in\left[B_{\tau}\right]^{n}\right\}$. Then $\left|\bar{B}_{\tau}\right|=\beta_{i}$. So w.l.o.g. we can assume that, for every $\tau_{1}, \tau_{2}<\delta, \bar{B}_{\tau_{1}}=\bar{B}_{\tau_{2}}$. But then $q_{\tau_{1}} \approx q_{\tau_{2}}$ for every $\tau_{1}, \tau_{2}<\delta$. Since $q_{\tau_{1}} \uparrow \alpha_{\xi-1}=q_{\tau_{2}} \uparrow \alpha_{\xi-1}$ it is enough to show only that $q_{\tau_{1}}(2, i) \approx q_{\tau_{2}}(2, i)$. Let $q_{1}^{\prime} \in \mathscr{P}\left(\alpha_{\nu_{i}}, \alpha_{\nu_{i}+1}\right)$ be stronger than $q_{\tau_{1}}(2, i)$. Say $q_{1}^{\prime}=q_{\tau_{1}}(2, i)^{\wedge}\left\langle Q_{11}, \ldots, Q_{1 n}, B_{1}\right\rangle$. Then there are $\left\langle Q_{21}, \ldots, Q_{2 n}\right\rangle \in\left[B_{\tau_{2}}\right]^{n}$ s.t. $Q_{2 j} \cap$ $\beta_{i}=Q_{1 j} \cap \beta_{i}$ for every $j, 1 \leqslant j \leqslant n$. Let

$$
q_{2}^{\prime}=q_{\tau_{2}}(2, i)^{\wedge}\left\langle Q_{21}, \ldots, Q_{2 n}, B_{2}\right\rangle,
$$

where

$$
B_{2}=\left(B_{\tau_{2}} \cap B_{1}-\left\{Q \in \mathscr{P}_{\alpha_{\nu_{i}}}\left(\alpha_{\nu_{i}+1}\right) \mid Q \nsupseteq Q_{2 n} \text { or } Q \cap \alpha_{\nu_{i}} \leqslant Q_{2 n} \cap \alpha_{\nu n_{i}}\right\}\right) .
$$

Then $\bar{q}_{1}^{\prime}=\bar{q}_{1}^{\prime} \cap B_{2}$ is similar to $q_{2}^{\prime}$. In the same way it is possible to find every $q_{2}^{\prime} \geqslant q_{\tau_{2}}(2, i)$ and $q_{1}^{\prime} \geqslant q_{\tau_{1}}(2, i)$ s.t. a direct extension of $q_{2}^{\prime}$ is similar to $q_{1}^{\prime}$. Hence $q_{\tau_{1}}(2, i)$ and $q_{\tau_{2}}(2, i)$ are almost similar, and so $q_{\tau_{1}}, q_{\tau_{2}}$ are almost similar.

The case $m_{2}<i \leqslant m_{3}$ is as the Prikry case above.

Case 3. $\xi$ is a limit ordinal. Let $Q$ that appears in $p \mid \mathbf{R}$ be s.t. $Q \cap \kappa=\alpha_{\xi}$. Let $\delta=\left(2^{2^{C} Q^{(\beta(Q))+}}\right)$, where $\beta(Q)$ is as in Theorem 3.2.10. Let $\left\{q_{\tau} \mid \tau<\delta\right\} \subseteq \overline{\mathbf{P}} \uparrow \alpha_{\xi}$. Let $Q_{1}$ be the maximal limit point of $p \uparrow \mathbf{R}$ below $Q$, if such exists, or the empty set otherwise. Let $Q_{1} \cap \kappa=\gamma$. For every $i, 1 \leqslant i \leqslant m_{3}, \alpha_{\nu_{i}}<\alpha_{\xi}$ implies $\alpha_{\nu_{i}} \leqslant \gamma$ since $p \in D\left(\nu_{i}+2\right)$. So between $\gamma$ and $\alpha_{\xi}$ we have only the supercompact Radin forcing. Since the cardinality of $\overline{\mathbf{P}} \uparrow \gamma$ is less than $\delta$, it is safe to assume that $q_{\tau_{1}} \uparrow \gamma=q_{\tau_{2}} \uparrow \gamma$ 
for every $\tau_{1}, \tau_{2}<\delta$. For $\tau<\delta$ let

$$
\bar{q}_{\tau}=\left\{\left\langle Q_{1}, u_{0}, B_{\tau_{0}}\right\rangle,\left\langle P_{\tau 1}, u_{\tau 1}, B_{\tau 1}\right\rangle, \ldots,\left\langle P_{\tau n_{\tau}}, u_{\tau n_{\tau}}, B_{\tau n_{\tau}}\right\rangle,\left\langle Q, u, B_{\tau}\right\rangle\right\}
$$

be the part of $q_{\tau} \uparrow \mathbf{R}$ between $Q_{1}$ and $Q$. W.l.o.g. we can assume that, for every $\tau_{1}, \tau_{2}<\delta$,

(a) $n_{\tau_{1}}=n_{\tau_{2}}=n$,

(b) $P_{\tau_{1} j} \cap \beta(Q)=P_{\tau_{2} j} \cap \beta(Q)$;

for every $j, 1 \leqslant j \leqslant n$,

(c) $\left\langle u_{\tau_{1} j}, B_{\tau_{1} 1}\right\rangle=\left\langle u_{\tau_{2} j}, B_{\tau_{2} j}\right\rangle=\left\langle u_{j}, B_{j}\right\rangle$;

and for every $j, 1 \leqslant j \leqslant n$,

(d) $B_{\tau_{1} 0}=B_{\tau_{2} 0}=B_{0}$.

We denote by $\bar{B}_{\tau}$ the set $\left\{\left\langle\left\langle T_{1} \cap \beta(Q), v_{1}, E_{1}\right\rangle, \ldots,\left\langle T_{m} \cap \beta(Q), v_{m}, E_{m}\right\rangle\right\rangle \mid m \in \omega\right.$, $\left\langle\left\langle T_{1}, v_{1}, E_{1}\right\rangle, \ldots,\left\langle T_{m}, v_{m}, E_{m}\right\rangle\right\rangle$ is addable to $\left.\left\langle Q, u, B_{\tau}\right\rangle\right\}$. The number of such $\bar{B}_{\tau}$ 's is less than $\delta$. So let us assume that, for every $\tau_{1}, \tau_{2}<\delta, \bar{B}_{\tau_{1}}=\bar{B}_{\tau_{2}}$. But then every two $q_{\tau_{1}}, q_{\tau_{2}}$ will be almost similar. It is enough to show only that $q_{1}={ }_{\mathrm{df}} q_{\tau_{1}}$ i $\mathbf{R} \backslash\left(P_{\tau_{1} n} \cap \kappa\right)$ and $q_{2}={ }_{\mathrm{df}} q_{\tau_{2}} \uparrow \mathbf{R} \backslash\left(P_{\tau_{2} n} \cap \kappa\right)$ are almost similar. So let

$$
q_{1}^{\prime}=q_{1} \uparrow\left\{\left\langle T_{1}, v_{1}, E_{1}\right\rangle, \ldots,\left\langle T_{m}, v_{m}, E_{m}\right\rangle\right\} .
$$

Then $\left\{\left\langle T_{1} \cap \beta(Q), v_{1}, E_{1}\right\rangle, \ldots,\left\langle T_{m} \cap \beta(Q), v_{m}, E_{m}\right\rangle\right\} \in \bar{B}_{\tau_{1}}=\bar{B}_{\tau_{2}}$. Hence there is a sequence $\left\{\left\langle S_{1}, v_{1}, E_{1}\right\rangle, \ldots,\left\langle S_{m}, v_{m}, E_{m}\right\rangle\right\}$ addable to $\left\langle Q, u, B_{\tau_{2}}\right\rangle$ such that $S_{j} \cap$ $\beta(Q)=T_{j} \cap \beta(Q)$ for every $j, 1 \leqslant j \leqslant m$.

Let $\bar{q}_{1}^{\prime}=q_{1}^{\prime} \frown\left(B_{\tau_{1}} \cap B_{\tau_{2}}\right)$ and $q_{2}^{\prime}=q_{2} \frown\left\{\left\langle S_{1}, v_{1}, E_{1}\right\rangle, \ldots,\left\langle S_{m}, v_{m}, E_{m}\right\rangle\right\}$. Then $\bar{q}_{1}^{\prime}$ is a direct extension of $q_{1}^{\prime}, \bar{q}_{1}^{\prime}, q_{2}^{\prime}$ are similar and they satisfy the conditions of Theorem 3.2.10. This completes the proof of Lemma 3.2.15.

Now we return to the proof of Theorem 3.2.11.

Let $l_{1} \leqslant M$ be the least s.t. $\alpha\left(l_{1}\right) \geqslant \alpha_{\nu_{i}}$ for $i \in\left[1, m_{2}\right]$, i.e. above $\alpha\left(l_{1}\right)$ in $\overline{\mathbf{P}}$ everything comes from R. For every $\gamma<\alpha_{\nu}$ and every $q \in \mathbf{P} \uparrow \alpha\left(l_{1}\right)$, Woodin's argument [17] provides a sequence $\vec{C}(\gamma, q)$ of sequences of sets of measure one above $\alpha\left(l_{1}\right)$ so that the following holds. There is $q^{\prime} \geqslant q^{\wedge} p \backslash \alpha\left(l_{1}\right)^{\wedge} \vec{C}(\gamma, q), q^{\prime} \uparrow$ $\alpha\left(l_{1}\right)=q$ and

$$
q^{\prime} \Vdash^{i} \Psi(\check{\gamma}) \quad \text { iff } \quad q^{\wedge} p \backslash \alpha\left(l_{1}\right)^{\frown} \vec{C}(\gamma, q) \Vdash^{i} \Psi(\check{\gamma}),
$$

where $i \in 2$ and ${ }^{0} \Psi=\Psi,{ }^{1} \Psi=\neg \Psi$, but for $q_{1} \approx q_{2}, \vec{C}\left(\gamma, q_{1}\right)=\vec{C}\left(\gamma, q_{2}\right)$ which follows from Woodin's construction of $\vec{C}(\gamma, q)$ and Lemma 3.2.13. Using completeness of measures above $\alpha\left(l_{1}\right)$ and Lemma 3.2 .15 we can replace all $\vec{C}(\gamma, q)$ by a single set $\vec{C}$.

Then, for every $q \in \overline{\mathbf{P}} \mid \alpha\left(l_{1}\right)$ and $\gamma<\alpha_{\nu}$, there is $q_{1} \in \overline{\mathbf{P}} \mid \alpha\left(l_{1}\right)$ s.t. $q_{1} \geqslant q$ and

$$
q_{1} \uparrow p \backslash \alpha\left(l_{1}\right) \frown \vec{C} \| \Psi(\check{\gamma}),
$$

since there is some $q^{\prime} \geqslant q^{\wedge} p \backslash \alpha\left(l_{1}\right)^{\wedge} \vec{C}$ which decides $\Psi(\check{\gamma})$. Let $q_{1}=q^{\prime} \mid \alpha\left(l_{1}\right)$. Then $q_{1} \wedge p \backslash \alpha\left(l_{1}\right)^{\wedge} \vec{C}\left(\gamma, q_{1}\right) \| \Psi(\check{\gamma})$. But by choice of $\vec{C}, q_{1} \wedge p \backslash \alpha\left(l_{1}\right)^{\wedge} \vec{C}$ is stronger than $q_{1} \frown p \backslash \alpha\left(l_{1}\right) \frown \vec{C}\left(\gamma, q_{1}\right)$.

Now let us define the sets $\vec{t}(l)$ by induction for every $l \leqslant l_{1}$ such that

(1) $p \backslash \alpha(l)^{\sim} \vec{t}(l) \in \overline{\mathbf{P}} \backslash \alpha(l)$,

(2) for every $q \in \overline{\mathbf{P}} \mid \alpha(l)$ and every $\gamma<\alpha_{\nu}$, there is $q^{\prime} \in \overline{\mathbf{P}} \mid \alpha(l)$ s.t. $q^{\prime} \geqslant q$ and

$$
q^{\prime} p \backslash \alpha(l) \frown \vec{t}(l) \| \Psi(\check{\gamma}) .
$$


Set $\vec{t}\left(l_{1}\right)=\vec{C}$. Suppose now that $\vec{t}\left(l^{\prime}\right)$ is defined for every $l^{\prime}, l<l^{\prime} \leqslant l_{1}$. Let us define $\vec{t}(l)$. Suppose $\alpha(l)=\alpha_{\xi}$ for some $\xi<\kappa$. We consider two cases.

Case 1. $\alpha(l+1)=\alpha_{\nu_{i}}$ for some $i, m_{1}<i \leqslant m_{4}$. So Prikry or Radin forcings were used between $\alpha_{\xi+1}$ and $\alpha(l+1)$. By [13 or 17] for every $q \in \overline{\mathbf{P}} \uparrow \alpha(l)$ there is a sequence of sets, $\vec{C}(\gamma, q)$, of measure one above $\alpha(l)$, so that the following holds. There is $q^{\prime} \geqslant q^{\wedge} p \backslash \alpha(l)^{\frown} \vec{t}_{\gamma}(l+1)^{\frown} \vec{C}(\gamma, q), q^{\prime} \uparrow \alpha(l)=q$,

$$
q^{\prime} \Vdash{ }^{i} \Psi(\check{\gamma}) \quad \text { iff } \quad q^{\wedge} p \backslash \alpha(l) \wedge \vec{t}_{\gamma}(l+1) \wedge \vec{C}(\gamma, q) \Vdash^{i} \Psi(\check{\gamma}) \quad \text { for } i \in 2 .
$$

Once more, for $q_{1} \approx q_{2}, \vec{C}\left(\gamma, q_{1}\right)=\vec{C}\left(\gamma, q_{2}\right)$ which follows from the construction of $\vec{C}(\gamma, q)$ in [13 or 17] and Lemma 3.2.13. By completeness of measures and Lemma 3.2.15 it is possible to replace all $\vec{C}(\gamma, q)$ by its coordinatewise intersection $\vec{C}$. Set $\vec{t}(l)=\vec{C} \wedge \vec{t}(l+1)$. Then, clearly, $p \backslash \alpha(l)^{\wedge} \vec{t}(l) \in \overline{\mathbf{P}} \backslash \alpha(l)$. Let $q \in \overrightarrow{\mathbf{P}} \backslash \alpha(l)$, $\gamma<\alpha_{\nu}$. By the inductive assumption there is $q^{\prime \prime} \in \mathbf{P} \uparrow \alpha(l+1)$ s.t. $q^{\prime \prime} \geqslant q^{\wedge} p \uparrow$ $\alpha(l+1)^{\wedge} \vec{C}$ and

$$
q^{\prime \prime} p \backslash \alpha(l+1) \wedge \vec{t}(l+1) \| \Psi(\check{\gamma}) .
$$

Set $\bar{q}^{\prime}=q^{\prime \prime} p \backslash \alpha(l+1)^{\wedge} \vec{t}(l+1)$ and $q^{\prime}=\bar{q}^{\prime} \uparrow \alpha(l)$. Then

$$
\bar{q}^{\prime} \geqslant q^{\prime} p \backslash \alpha(l) \wedge \vec{t}_{\gamma}(l+1) \frown \vec{C} .
$$

It implies that $\bar{q}^{\prime} \geqslant q^{\prime} p \backslash \alpha(l)^{\wedge} \vec{t}_{\gamma}(l+1)^{\frown} \vec{C}\left(\gamma, q^{\prime}\right)$. Hence

$$
q^{\prime} p \backslash \alpha(l) \frown \vec{t}_{\gamma}(l+1) \frown \vec{C}(\gamma, q) \| \Psi(\check{\gamma}) .
$$

So the stronger condition $q^{\prime \wedge} p \backslash \alpha(l)^{\wedge} \vec{t}_{\gamma}(l)$ decides $\Psi(\check{\gamma})$, but $q^{\prime} \geqslant q$. Case 1 is proved.

Case 2. $\alpha(l+1)=\alpha_{\nu_{i}}$ for some $i, 1 \leqslant i \leqslant m_{1}$. Then $\nu_{i}$ is a nonlimit ordinal and the Levy collapse was used between $\alpha_{\nu_{i}}$ and $\alpha_{\nu_{i}+1}$. Let us assume for simplicity that $\alpha_{\nu_{i}-1}=\alpha(l)$. If $\alpha_{\nu_{i}-1}>\alpha(l)$, then we shall first go down to $\overline{\mathbf{P}} \mid \alpha_{\nu_{i}-1}$ and then continue as in Case 1 to $\mathbf{P} \mid \alpha(l)$. Let $T$ be a maximal subset of $\bar{P} \mid \alpha(l)$ consisting of pairwise not almost similar elements.

Then by Lemma 3.2.15 the cardinality of $T$ is some $\delta<\alpha_{\nu_{i}}=\alpha(l+1)$. Let $\left\langle q_{\tau}\right| \tau\langle\delta\rangle$ be an enumeration of $T$. We define by induction an increasing sequence $\langle r(\gamma)| \gamma\left\langle\alpha_{\nu}\right\rangle$ of conditions in $\operatorname{Col}\left(\alpha_{\nu_{i}}, \alpha_{\nu_{i}+1}\right)$. Let $r(0)=p(1, i)$. Suppose $\left\langle r\left(\gamma^{\prime}\right) \mid \gamma^{\prime}<\gamma\right\rangle$ is defined. Let $\tilde{r}=\bigcup\left\{r\left(\gamma^{\prime}\right) \mid \gamma^{\prime}<\gamma\right\} . r(\gamma)$ will be the union of the seauence $\left\langle s_{\tau}\right| \tau\langle\delta\rangle$ defined as follows. Set $s_{0}=\tilde{r}$. Now if there exists an $s \in$ $\operatorname{Col}\left(\alpha_{\nu_{i}}, \alpha_{\nu_{i}+1}\right), s \geqslant r$, s.t.

$$
q_{0} s^{\wedge} p \backslash \alpha(l+1) \wedge \vec{t}(l+1) \| \Psi(\check{\gamma}),
$$

then let $s_{1}=s$. Otherwise $s_{1}=s_{0}$. Suppose that, for every $\tau^{\prime}<\tau, s_{\tau^{\prime}}$ is defined. Let $s_{\tau}^{\prime}=\bigcup\left\{s_{\tau^{\prime}} \mid \tau^{\prime}<\tau\right\}$. Notice that $\delta<\alpha_{\nu_{i}}$ so $s_{\tau}^{\prime} \in \operatorname{Col}\left(\alpha_{\nu_{i}}, \alpha_{\nu_{i}+1}\right)$. If there exists an $s \geqslant s_{\tau}^{\prime}$ s.t.

$$
q_{\tau} s^{\wedge} p \backslash \alpha(l+1) \frown \vec{t}(l+1) \| \Psi(\check{\gamma}),
$$

then let $s_{\tau}=s$. Otherwise set $s_{\tau}=s_{\tau}^{\prime}$.

Now set $r=\bigcup\left\{r(\gamma) \mid \gamma<\alpha_{\nu}\right\}$. Since $l+1>0, \alpha_{\nu}<\alpha_{\nu_{i}}=\alpha(l+1)$ and so $r \in$ $\operatorname{Col}\left(\alpha_{\nu_{i}}, \alpha_{\nu_{i}+1}\right)$. We define $\vec{t}(l)=r^{\sim} \vec{t}(l+1)$. 
Let us check that for every $q \in \overline{\mathbf{P}} \uparrow \alpha(l)$ and $\gamma<\alpha_{\nu}$ there is $q^{\prime} \in \overline{\mathbf{P}} \uparrow \boldsymbol{\alpha}(l)$ s.t. $q^{\prime} \geqslant q$ and $q^{\prime \wedge} p \backslash \alpha(l)^{\wedge} \vec{t}(l) \| \Psi(\check{\gamma})$. So, for fixed $q \in \overline{\mathbf{P}} \uparrow \alpha(l+1)$ and $\gamma<\alpha_{\nu}$, let $q^{\prime \prime} \in \overline{\mathbf{P}} \mid \alpha(l+1)$ be s.t. $q^{\prime \prime} \geqslant q^{\wedge} r$ and $q^{\prime \prime} p \backslash \alpha(l+1)^{\wedge} \vec{t}(l+1) \| \Psi(\check{\gamma})$. Set $q^{\prime}=q^{\prime \prime}$ i $\alpha(l)$. Let $q^{\prime \prime}=q^{\prime \wedge} s$ for some $s \in \operatorname{Col}\left(\alpha_{\nu_{i}}, \alpha_{\nu_{i}+1}\right)$. Then $q^{\prime \prime} \approx q_{\tau}$ for some $\tau<\delta$. By Lemma 3.2.13,

$$
q_{\tau}{ }^{\wedge} p \backslash \alpha(l+1) \frown \vec{t}(l+1) \| \Psi(\gamma) .
$$

Then, on the step $\tau$ in the construction of $r(\gamma)$ we have

$$
q_{\tau}{ }^{\wedge} s_{\tau} p \backslash \alpha(l+1) \frown \vec{t}(l+1) \| \Psi(\gamma) .
$$

Hence $q_{\tau}{ }^{\wedge} r^{\wedge} \backslash \alpha(l+1)^{\wedge} \vec{t}(l+1) \| \Psi(\gamma)$ since $r \geqslant r(\gamma) \geqslant s_{\tau}$. Applying Lemma 3.2.13, we obtain that $q^{\prime} r^{\wedge} p \backslash \alpha(l+1)^{\wedge} \vec{t}(l+1) \| \Psi(\gamma)$. But it means that

$$
q^{\prime} p \backslash \alpha(l) \frown \vec{t}(l) \| \Psi(\gamma) .
$$

Case 2 is proved.

Now for $l=0$ we have a set $\vec{t}(0)$ s.t., for every $q \in \overline{\mathbf{P}} \mid \alpha_{\nu}$, every $\gamma<\alpha_{\nu}$ and every $\gamma<\alpha_{\nu}$, there is $q^{\prime} \in \overline{\mathbf{P}} \mid \alpha_{\nu}$ s.t. $q^{\prime} \geqslant q$ and $\left.q^{\prime} \cap \mathrm{p} \backslash \alpha_{\nu}\right\urcorner \vec{t}(0) \| \Psi(\gamma)$, where the $p$ that we started with can be chosen as an extension of an arbitrary condition. The above means that $\overline{\mathbf{P}} \mid \alpha_{\nu}$ already determines the set $a$. This completes the proof of Theorem 3.2.11(i).

(ii) It is enough proof that $\alpha_{\nu}$ is a cardinal in $\operatorname{HMD}(A(G))$ only for nonlimit $\nu$. So let $\nu=\nu^{\prime}+1$. Now if $\alpha_{\nu}$ is collapsed, there must be a function $f: \alpha_{\nu^{\prime}} \leftrightarrow \alpha_{\nu}$, by Lemma 3.2.1. It can be coded as a subset of $\alpha_{\nu^{\prime}}$. Hence $f \in M\left[G \mid \alpha_{\nu^{\prime}}\right]$. But it is impossible, since by Lemmas 3.2.13 and 3.2.15, almost similar conditions in $\mathbf{P} \mid \boldsymbol{\alpha}_{\nu^{\prime}}$ must force the same values and there are less than $\alpha_{\nu}$ pairwise not almost similar conditions. This completes the proof of Theorem 3.2.11(ii).

(iii) Suppose now that $\nu+1 \in A$ and there is $f: \alpha_{\nu} \rightarrow \alpha_{\nu+1}$ unbounded in $\alpha_{\nu+1}$.

Let $f$ be determined by some formula $\varphi\left(x_{0}, x_{1}, \ldots, x_{n+2}\right)$ which include some parameters from $M$, some $i_{G}\left(\pi_{1} x_{\nu_{1}}\left(\beta_{1}\right)\right), \ldots, i_{G}\left(\pi_{n} x_{\nu_{n}}\left(\beta_{n}\right)\right)$, and $A(G)$, i.e. for $\alpha<\alpha_{\nu}$, $\beta<\alpha_{\nu+1}$

$$
f(\alpha)=\beta \quad \text { iff } \quad M[G]=\varphi\left(\alpha, \beta, i_{G}\left(\pi_{1} \underline{x}_{\nu_{1}}\left(\beta_{1}\right)\right), \ldots, A(G)\right)={ }_{\mathrm{df}} \Psi(\alpha, \beta) .
$$

Let us use the same notation as in (i). Then in (i), there is some $\vec{t}$ s.t.

(1) $p \backslash \alpha_{\nu+1} \bumpeq \vec{t} \in \overline{\mathbf{P}} \backslash \alpha_{\nu+1}$,

(2) for every $q \in \overline{\mathbf{P}} \uparrow \alpha_{\nu+1}$ and every $\gamma_{1}<\alpha_{\nu}, \gamma_{2}<\alpha_{\nu+1}$, there is $q^{\prime} \in \overline{\mathbf{P}} \uparrow \alpha_{\nu+1}$ s.t. $q^{\prime} \geqslant q$ and

$$
q^{\prime} p \backslash \alpha_{\nu+1} \wedge \vec{t} \| \Psi\left(\check{\gamma}_{1}, \check{\gamma}_{2}\right) .
$$

Let $\left\langle q_{\tau} \mid \tau<\delta\right\rangle$ be a maximal subset of $\overline{\mathbf{P}} \uparrow \alpha_{\nu}$ consisting of pairwise not almost similar conditions. By Lemma 3.2.15, $\delta<\alpha_{\nu+1}$. By induction we define, for every $\gamma<\alpha_{\nu}$, an increasing sequence $\left\langle r(\gamma) \mid \gamma<\alpha_{\nu}\right\rangle$ of conditions in $\operatorname{Col}\left(\alpha_{\nu+1}, \alpha_{\nu+2}\right)$ as follows. Notice that this forcing was used for $\alpha_{\nu+1}$ since $\nu+1 \in A$. Let $r(0)=$ $\left(p \uparrow \alpha_{\nu+1}\right) \backslash \alpha_{\nu}$. Suppose that $\left\langle r\left(\gamma^{\prime}\right) \mid \gamma^{\prime}<\gamma\right\rangle$ is defined. Set $\bar{r}=\bigcup\left\{r\left(\gamma^{\prime}\right) \mid \gamma^{\prime}<\gamma\right\}$. $r(\gamma)$ will be the union of the increasing sequence $\left\langle s_{\tau} \mid \tau<\delta\right\rangle$ defined below. Set $s_{0}=\bar{r}$. Now if there are some $s \geqslant s_{0}$ and $\beta<\alpha_{\nu+1}$ s.t. $q_{0}^{\wedge} s^{\wedge} p \backslash \alpha_{\nu+1} \wedge \vec{t} \Vdash \Psi(\check{\gamma}, \breve{\beta})$, 
then let $s_{1}=$ some such $s$. Otherwise $s_{1}=s_{0}$. We continue in such a way for every $\tau<\delta$, i.e. if there are $s \geqslant s_{\tau}$ and $\beta<\alpha_{\nu+1}$ s.t.

$$
\left.q_{\tau} s^{\wedge} p \backslash \alpha_{\nu+1}\right\urcorner \vec{t} \Vdash \Psi(\check{\gamma}, \check{\beta}),
$$

then we pick some such $s$ and set $s_{\tau+1}=s$. Otherwise $s_{\tau+1}=s_{\tau}$. It completes the definition of $\left\langle r(\gamma) \mid \gamma<\alpha_{\nu}\right\rangle$. Set $r=\bigcup_{\gamma<\alpha_{\nu}} r(\gamma)$. Then the condition

$$
p \nmid \alpha_{\nu} \wedge r^{\wedge} p \backslash \alpha_{\nu+1} \wedge \vec{t}
$$

forces that the function $\Psi$ be bounded in $\alpha_{\nu+1}$ and, by Lemma 3.2.13, almost similar conditions force the same. This completes the proof of Theorem 3.2.11(iii).

The next statement based on Mitchell's result [12] says that the regularity of $\kappa$ is preserved in generic extensions by Radin forcing of the length $\kappa^{+}$. In our case only one additional argument is needed.

THEOREM 3.2.16. $\kappa$ is a regular cardinal in $\mathrm{HM} \mathrm{D}(A(G))$.

Proof. Suppose otherwise. Then in $M[G]$ there is an $M$-definable function $\sigma$ with parameters from $A(G)$ from some $\delta<\kappa$ unboundedly into $\kappa$. Let

$$
\varphi\left(x_{0}, x_{1}, i_{G}\left(\pi_{1} x_{\nu_{1}}\left(\beta_{1}\right)\right), \ldots, i_{G}\left(\pi_{n} \underline{x_{\nu_{n}}\left(\beta_{n}\right)}\right), A(G)\right)
$$

be a formula which defines $\sigma$ and is allowed to also contain some parameters from $M$. Let us denote $\varphi$ by $\Psi\left(x_{0}, x_{1}\right)$.

Let $p$ and $\overline{\mathbf{P}}$ be as in Theorem 3.2.11 for the formula $\Psi$. Suppose also that, for some $\nu>\operatorname{Max}\left\{\nu_{i} \mid 1 \leqslant i \leqslant n\right\}, \alpha_{\nu}$ appears in $p$. Let $Q$ be in $p$ s.t. $Q \cap \kappa=\alpha_{\nu}$.

LeMma 3.2.17. Let $\left\langle q_{\alpha}\right| \alpha\left\langle\kappa^{+}\right\rangle$be in $\bar{P}$ and let every $q_{\alpha}$ be of the form $\left.p_{\alpha}\right\urcorner\left\langle M_{<\kappa^{+}}, T\right\rangle$, for some fixed $T$, where every $p_{\alpha}$ is a lower part, i.e. there is no $M_{<\kappa^{+}}$ in $p_{\alpha}$.

Then there are $\tau_{1}, \tau_{2}<\kappa^{+}$s.t. $q_{\tau_{1}}, q_{\tau_{2}}$ are similar and satisfy the conditions (1)-(4) of Theorem 3.2.10.

Proof. W.l.o.g. we can assume that $p_{\tau_{1}} \uparrow \alpha_{\nu}=p_{\tau_{2}} \uparrow \alpha_{\nu}$ for every $\tau_{1}, \tau_{2}<\kappa^{+}$, since $\alpha_{\nu}$ appears in $p$, and so $\left|\mathbf{P} \uparrow \alpha_{\nu}\right|<\kappa$. Let

$$
p_{\tau} \backslash \alpha_{\nu}=\left\{\left\langle Q, u, B_{\tau 0}\right\rangle,\left\langle Q_{\tau 1}, u_{\tau 1}, B_{\tau 1}\right\rangle, \ldots,\left\langle Q_{\tau m_{\tau}}, u_{\tau m_{\tau}}, B_{\tau m_{\tau}}\right\rangle\right\} .
$$

It is safe to assume that for every $\tau_{1}, \tau_{2}<\kappa^{+}$

(a) $m_{\tau_{1}}=m_{\tau_{2}}=m$,

(b) $P_{\tau_{1} j} \cap \kappa=P_{\tau_{2} j} \cap \kappa$ for every $j, 1 \leqslant j \leqslant m$,

(c) $\left\langle u_{\tau_{1} j}, B_{\tau_{1} j}\right\rangle=\left\langle u_{\tau_{2} j}, B_{\tau_{2} j}\right\rangle$ for every $j, 1 \leqslant j \leqslant m$,

(d) $B_{\tau_{1} 0}=B_{\tau_{2} 0}$.

But (a)-(d) together with $p_{\tau_{1}} \uparrow \alpha_{\nu}=p_{\tau_{2}} \uparrow \alpha_{\nu}$ means that $q_{\tau_{1}}, q_{\tau_{2}}$ are similar and satisfy conditions (1)-(4) of Theorem 3.2.10.

The continuation of the proof is as in Mitchell [12]. Lemma 3.2.15 and Theorem 3.2.10 are used to insure that the set $\{\nu(\xi, n, p) \mid \xi<\delta, n<\omega, p$ is a lower part addable to $\left.\left\langle M_{<\kappa^{+}}, T\right\rangle\right\}$ is bounded below $\kappa^{+}$. 
4. The model $N_{A}$.

Definition 4.1. Let $N_{A}$ be the set of all sets in $\mathrm{HMD}(A(G))$ of rank less than $\kappa$.

THEOREM 4.2. $N_{A}$ is a model of $Z F$.

Proof. Obviously, since $\kappa$ is a limit cardinal in $\operatorname{HMD}(A), N_{A}$ satisfies all the axioms of ZF except probably the Replacement Axiom.

Suppose that it fails in $N_{A}$. Then for some set $X$ in $N_{A}$ there is a class function $F$ on $X$ s.t. $F^{\prime \prime}(X) \notin N_{A}$. Since $N_{A}$ satisfies the Separation Axiom, w.1.o.g. we can assume that $\operatorname{rng} F \subseteq \kappa$ and it is unbounded in $\kappa$. Also let $X=V_{\alpha}$ for some $\alpha<\kappa$ and let $\alpha$ be the minimal s.t. there is a class function from $V_{\alpha}$ unboundedly into $\kappa$. Such $\alpha$ cannot be a limit ordinal since then there is a class function $F^{\prime}: \alpha \rightarrow \kappa$, unbounded in $\kappa$. But $\mathrm{H} M \mathrm{D}(A(G))$ is a model of $\mathrm{ZF}$, hence $F^{\prime} \in \mathrm{H} M \mathrm{D}(A(G))$ and so, $\kappa$ is a singular cardinal in $\mathrm{H} M \mathrm{D}(A(G))$. It contradicts Theorem 3.2.16. So, for some $\delta<\kappa$, there is, in $\operatorname{HMD}(A(G)), F: \mathscr{P}\left(V_{\delta}\right) \rightarrow \kappa$ s.t. rng $F$ is unbounded in $\kappa$. In order to complete the proof we need the following lemma.

Lemma 4.3. Let $\beta=\beta^{\prime}+n$ for a limit ordinal $\beta^{\prime}<\kappa$. Then

$$
V_{\beta}^{N_{A}} \subseteq V_{\beta}^{M\left[G \mid \alpha_{\left.\beta^{\prime}+2 n\right]}\right.}
$$

Proof. We prove the lemma by induction on $\beta$.

If $\beta$ is limit and the statement holds for every $\gamma<\beta$, then

$$
\begin{aligned}
& V_{\beta}^{N_{A}}=\bigcup_{\gamma<\beta} V_{\gamma}^{N_{A}} \subseteq \bigcup\left\{V_{\gamma}^{M\left[G\left[\alpha_{\gamma^{\prime}+2 n}\right]\right.} \mid \gamma<\beta, \gamma=\gamma^{\prime}+n\right. \\
& \text { for some } \left.n<\omega \text { and } \gamma^{\prime}<\beta \text { a limit ordinal }\right\} \\
& \subseteq \bigcup\left\{V_{\gamma}^{M\left[G \mid \alpha_{\beta}\right]} \mid \gamma<\beta\right\}=V_{\beta}^{M\left[G\left\lceil\alpha_{\beta}\right]\right.} \text {. }
\end{aligned}
$$

Now suppose $\beta=\beta^{\prime}+n$ for a limit ordinal $\beta^{\prime}<\kappa, n \neq 0$ and

$$
V_{\beta^{\prime}+n-1}^{N_{A}} \subseteq V_{\beta^{\prime}+n-1}^{M\left[G \mid \alpha_{\beta^{\prime}+2(n-1)}\right]} \text {. }
$$

The cardinality of $V_{\beta^{\prime}+n-1}^{M\left[G \mid \alpha_{\beta^{\prime}+2(n-1)}\right]}$ is less than $\alpha_{\beta^{\prime}+2 n}$ in $M\left[G \mid \alpha_{\beta^{\prime}+2(n-1)}\right]$ since $\alpha_{\beta^{\prime}+2 n}$ is inaccessible in this model. Let $a \subseteq V_{\beta^{\prime}+n-1}^{N_{A}}$ be in $N_{A}$. Then $a$ can be viewed as a subset of $\alpha_{\beta^{\prime}+2 n}$ which belongs to $\mathrm{H} M\left[G \mid \alpha_{\beta^{\prime}+2(n-1)}\right] \mathrm{D}(A(G))$. Then the analog of Theorem 3.2.11, for $M$ replaced by $M\left[G \mid \alpha_{\beta^{\prime}+2(n-1)}\right]$, implies $a \in M\left[G \mid \alpha_{\beta^{\prime}+2 n}\right]$.

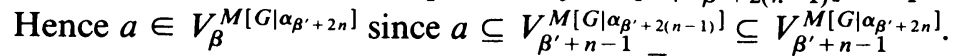

Now using the lemma, we can find $\bar{F}$ in $\mathrm{HM}\left[G \mid \alpha_{\bar{\delta}}\right] \mathrm{D}(A(G))$ s.t. $\bar{F}: \bar{\alpha} \rightarrow \kappa$ unboundedly for some $\bar{\alpha}<\kappa$, where $\bar{\delta}=\delta^{\prime}+2 n$ for the maximal limit $\delta^{\prime}<\delta$ s.t. $\delta=\delta^{\prime}+n$. But it contradicts the analog of Theorem 3.2.16 for $M$ replaced by $M\left[G \mid \alpha_{\bar{\delta}}\right]$. So we get the contradiction to the assumption that the Axiom of Replacement fails in $N_{A}$.

THEOREM 4.4. For every $\alpha>0, N_{A} \vDash \aleph_{\alpha}$ is regular iff $\alpha \in A$.

The proof follows from 3.2.1, 3.2.2 and 3.2.11. 


\section{Some open questions.}

Question 1. Is there a model $M$ of ZFC without weakly inaccessible cardinals s.t. for every class $A$ of $M$ consisting of nonlimit ordinals there is a model $N_{A}$ of ZF s.t. those regular alephs are exactly $\left\{\boldsymbol{\aleph}_{\alpha} \mid \alpha \in A \cup\{0\}\right\}$ ?

Notice that the model we started with has a lot of inaccessible cardinals below $\kappa$.

Now about the consistency strength: clearly, Con $\left(\mathrm{ZF}+\right.$ "only $\boldsymbol{\aleph}_{0}$ and $\boldsymbol{\aleph}_{1}$ are regular") implies $\operatorname{Con}\left(\mathrm{ZF}+\right.$ " $\forall \alpha>0 \boldsymbol{N}_{\alpha}$ is singular"). It is natural to ask the following

Question 2. Does Con(ZF $+\forall \alpha>0 \boldsymbol{\aleph}_{\alpha}$ is singular) imply Con(ZF + "only $\boldsymbol{\aleph}_{0}$ and $\aleph_{1}$ are regular alephs")?

Our conjecture is that the statement $\operatorname{Con}\left(\mathrm{ZF}+\right.$ "only $\boldsymbol{\aleph}_{0}$ and $\boldsymbol{\aleph}_{1}$ are regular alephs") is stronger. How strong is it? For example,

Question 3. Does Con(ZF + "only $\boldsymbol{\aleph}_{0}$ and $\boldsymbol{\aleph}_{1}$ are regular alephs") imply Con(ZF + "the regular uncountable alephs are exactly $\left.\left\{\boldsymbol{\aleph}_{\alpha+120} \mid \alpha \in 0 n\right\} "\right)$ ?

The following question may be more interesting.

Question 4. What are the possible values of the cofinality function in models of ZF?

Here we already do not know if it is possible to construct a model in which $\boldsymbol{\aleph}_{3}, \boldsymbol{\aleph}_{4}, \boldsymbol{\aleph}_{5}, \boldsymbol{\aleph}_{6}$ are all of the cofinality $\boldsymbol{\aleph}_{3}$.

If we take $\boldsymbol{\aleph}_{1}, \boldsymbol{\aleph}_{2}, \boldsymbol{\aleph}_{3}, \boldsymbol{\aleph}_{4}$ or $\boldsymbol{\aleph}_{2}, \boldsymbol{\aleph}_{3}, \boldsymbol{\aleph}_{4}, \boldsymbol{\aleph}_{5}$ instead, then it is possible from AD and we do not know any other way to obtain it. Our impression is that somewhere here must start statements provable in $\mathrm{ZF}$.

The next question is due to $\mathrm{H}$. Woodin and it is related to the previous one.

Question 5. Is there a model of $\mathrm{ZF}+\mathrm{DC}+$ "only $\boldsymbol{\aleph}_{0}$ and $\boldsymbol{\aleph}_{1}$ are the regular cardinals"?

In the models $N_{A}$ constructed above, already the Axiom of Choice for two-element sets fails.

Let us finish with the question this work started from. Unfortunately, we still do not know the answer.

Question 6 (Specker [16]). Is it consistent with ZF that, for every $\alpha, 2^{\kappa_{\alpha}}$ is a countable union of sets of cardinality $\boldsymbol{N}_{\alpha}$ ?

Feferman and Levy [3] proved that it is consistent with ZF + " $2{ }^{\aleph_{0}}$ is a countable union of countable sets". See also [5] for some related results.

ACKNOWLEDGEMENT. We are grateful to Hugh Woodin for many explanations of Radin forcing.

\section{REFERENCES}

1. A. Dodd, The core model, London Math. Soc. Lecture Notes Ser. 61, 1982.

2. W. Easton, Powers of regular cardinals, Ann. Math. Logic 1 (1970), 139-178.

3. S. Feferman and A. Levy, Independence results in set theory by Cohen's method. II, Notices Amer. Math. Soc. 10 (1963), 593.

4. M. Foreman and H. Woodin, The G.C.H. can fail everywhere (to appear).

5. M. Gitik, All uncountable cardinals can be singular, Israel J. Math. 35 (1980), 61-88.

6. _ـ_ Nonsplitting subset $\mathscr{P}_{\kappa}\left(K^{+}\right)$, J. Symbolic Logic (to appear).

7. T. Jech, Set theory, Academic Press, New York, 1978.

8. The axiom of choice, North-Holland, Amsterdam, 1973. 
9. A. Kanamori and M. Magidor, The evolution of large cardinal axioms in set theory, Lecture Notes in Math., vol. 669, Springer-Verlag, Berlin and New York, 1978, pp. 99-275.

10. M. Magidor, On the singular cardinals problem. I, Israel J. Math. 28 (1977), 1-31.

11. W. Mitchell, Hypermeasurable cardinals, Logic Colloq. 1978 (M. Boffa, D. Van Dalen and K. McAloon, eds.), North-Holland, Amsterdam, 1979, pp. 303-316.

12. __ How weak is a closed unbounded ultrafilter?, Logic Colloq 1980 (D. Van Dalen, D. Lascar and T. J. Smiley), North-Holland, Amsterdam, 1982, pp. 209-301.

13. K. Prikry, Changing measurable into accessible cardinals, Dissertationes Math. 68 (1970), 5-52.

14. L. Radin, Adding closed cofinal sequences to large cardinals, Ann. Math. Logic 3 (1982), 243-263.

15. R. Solovay, W. Reinhardt and A. Kanamori, Strong axioms of infinity and elementary embeddings, Ann. Math. Logic 13 (1978), 73-116.

16. E. Specker, Zur Axiomatik der Mengenlehre, Z. Math. Logik Grundlag. Math. 3 (1957), 173-210.

17. H. Woodin, The Prikry property for the Radin forcing, manuscript.

Department of Mathematics, California Institute of Technology, Pasadena, California 91125

Current address: School of Mathematical Sciences, Tel-Aviv University, Tel-Aviv, Israel 